\title{
Estrategias de innovación y dinámica del empleo en ramas productivas argentinas*
}

\author{
Verónica Robert, ${ }^{* *}$ Gabriel Yoguel, ${ }^{* * *}$ Luciano Cohan ${ }^{* * * *}$ y Luis Trajtenberg ${ }^{* * * * *}$
}

\section{RESUMEN}

Se analiza la relación entre desarrollo de capacidades, innovación y dinámica del empleo en una muestra de 403 empresas industriales con diferentes ramas productivas argentinas. Se parte de que el impacto de las actividades innovativas de las empresas sobre la dinámica del empleo no depende de los esfuerzos aislados sino de las estrategias de innovación, entendidas éstas como un conjunto de esfuerzos coordinados y coherentes, con los atributos sectoriales de la rama productiva en la que opera. En este sentido, se plantea una metodología en tres etapas: en la primera se propone identificar las estrategias de innovación, en la segunda se indaga el efecto de las capacidades en la determinación de las estrategias y la tercera aborda el impacto de las estrategias y capacidades sobre los cambios en el empleo. Los resultados del ejercicio econométrico planteado muestran que las capacidades de absorción y conectividad de las empresas repercuten en las estrategias de innovación de las empresas, mientras que éstas afectan la dinámica del empleo tanto en la cantidad de trabajadores como en sus calificaciones.

Palabras clave: estrategias de innovación, empleo, complejidad.

Clasificación JEL: B52, O30, J20.

\begin{abstract}
This article explores the relationship between capacity development, innovation and employment dynamics in a sample of 403 industrial firms form different production networks in Argentina. It begins with the idea that the impact of the firms' innovative activities on the dynamics of employment does not depend on isolated efforts but on innovation strategies, understood as a set of coordinated and consistent innovation efforts, once considered the sectoral attributes of the productive network in which the firm operates. We propose a methodology in three stages. First we propose the identification of innovation strategies, on the basis of innovation efforts performed, using a factor and cluster analysis. Secondly, we investigate the effect of absorption and connectivity capacities in determining the strategies using a multinomial regression. Lastly, we analyze the impact of strategies and capacities on employment dynamics. The results of the econometric exercises show that absorptive and connectivity capacity impact on firms' innovation strategies, while they affect the dynamics of employment in terms of number of workers and in terms of their qualifications.
\end{abstract}

Key words: innovation strategies, employment, complexity.

Classification JEL: B52, O30, J20.

* Una versión preliminar de este trabajo fue presentado en el Seminario EULAKS en Viena, en junio de 2009. Agradecemos los comentarios recibidos en esa ocasión, así como también las observaciones realizadas por los dictaminadores anónimos que ayudaron a mejorar el artículo.

** Investigadora del Instituto de Industria de la Universidad Nacional General Sarmiento, Buenos Aires: vrobert@gmail.com.*** Profesor titular de economía de la innovación, Universidad Nacional General Sarmiento: gyoguel@ungs.edu.ar **** Investigador del Instituto de Industria de la Universidad Nacional General Sarmiento: lucohan@yahoo.com.ar. ***** Profesor de estadística y econometría de la Universidad Nacional General Sarmiento: luis_trajtenberg@hotmail.com 


\section{INTRODUCCIÓN}

La relación entre la dinámica del empleo y la innovación ha sido motivo de preocupación de diferentes autores a lo largo de la historia del pensamiento económico. No obstante, a partir del profundo impacto que ha tenido la difusión de las nuevas tecnologías de la información y comunicación en los mercados laborales de todo el mundo, así como el creciente interés sobre el desempeño del cambio tecnológico en el crecimiento económico, este tema ha cobrado especial relevancia en las tres décadas pasadas. A pesar de los escasos consensos que pueden hallarse en muchas aportaciones a la problemática, hay un reconocimiento común de que la relación entre innovación y empleo es una relación compleja con múltiples factores implicados.

La bibliografía reciente sobre economía de la innovación ha mostrado algunos avances a partir de diversos estudios realizados en el ámbito de la empresa. Estos trabajos mostraron el impacto diferencial sobre la dinámica del empleo de los distintos resultados y esfuerzos de innovación (Stoneman, 1995; Pianta, 2006; Vivarelli, Evangelista y Pianta, 1996; Evangelista y Savona, 2001, Edquist, 2000, Petit, 1998; Brouwer et al., 1993; Tether, 2000). Así, por ejemplo, se ha podido establecer econométricamente que mientras las innovaciones de producto tienen mayoritariamente efectos positivos sobre el nivel de empleo de la empresa, las de proceso tienden a reducir el número de trabajadores. Del mismo modo, los diferentes esfuerzos de innovación incorporados (como adquisición de maquinaria) y desincorporados (como actividades de investigación y desarrollo) afectarían de diferentes formas tanto la cantidad como la calificación de los trabajadores de una empresa. En este sentido, esta rama de la bibliografía estuvo enfocada en identificar los factores asociados con el cambio tecnológico que tienen impacto positivo en la dinámica del empleo en el nivel de empresa.

Al mismo tiempo, diferentes autores evolucionistas han incluido un marco conceptual general inspirado en la teoría de los sistemas complejos para ex-plicar los procesos de aprendizaje, innovación y competencia en sistemas productivos y de innovación (Dosi, 1991; Dosi y Kaniovski, 1994; Dosi, y Nelson, 1994; Metcalfe, Foster y Ramlogan, 2006, Antonelli, 2008; Erbes, Robert y Yoguel, 2010, entre otros). De acuerdo con Erbes, Robert y Yoguel (2010) los resultados de innovación de las empresas no constituyen variables independientes sino que están determinados por el desarrollo de sus capacidades de absorción (Cohen y Levinthal, 1990) y conectividad (Grandori y Soda, 1995) y son una propiedad emergente del sistema productivo y de innovación en el que las 
empresas están inmersas. Esto obliga a replantear el alcance de enfoques analíticos que desmembran la actividad innovativa en un conjunto de esfuerzos, sin tener en cuenta las dinámicas no lineales de los procesos de aprendizaje. En esta dirección, Pianta (2006) propone que las decisiones de la empresa acerca de la modificación del número de empleados y/o su calificación, producto de las actividades de innovación, variarían de acuerdo con la estrategia de innovación seguida por la empresa más que por los esfuerzos o resultados tomados de forma aislada.

Desde una perspectiva teórica, este artículo combina el enfoque evolucionista con la teoría de los sistemas complejos para estudiar las estrategias de innovación y la dinámica del empleo asociada con la innovación en empresas que pertenecen a diferentes ramas productivas. En este sentido, interesa evaluar: i) la relación entre las capacidades de las empresas y las estrategias de innovación, y ii) el efecto de estas estrategias sobre las variaciones en la cantidad y la calificación de los trabajadores, asociadas con las actividades de innovación.

Se parte de la premisa de que la estrategia de innovación seguida por una empresa está compuesta por un conjunto de actividades de innovación, con mayor o menor grado de articulación. La elección de la estrategia de innovación de las empresas es afectada tanto por sus capacidades de absorción y de conectividad como por las características impresas en los regímenes sectoriales de innovación (Malerba y Orsenigo, 1997; Pavitt, 1984; Erbes et al. 2006), que, en consecuencia, constituirían las causas últimas de los cambios en la cantidad y calificación de la fuerza de trabajo asociadas con la innovación.

Desde una perspectiva metodológica, este artículo propone en primer lugar analizar el efecto de diferentes estrategias de innovación sobre la dinámica del empleo, en reemplazo de la metodología convencional que considera el impacto de los diferentes esfuerzos o resultados de innovación de forma aislada. Para esto, se identifican estrategias de innovación perseguidas por las empresas a partir de la conjunción de diversas actividades de innovación, sobre la base de un análisis de correspondencias múltiples y de clusters. En segundo lugar, siguiendo a Evangelista y Savona (2001), se considera la variación en el empleo asociada con las actividades de innovación y no con la evolución global del empleo. De esta forma se evitan los efectos del ciclo económico sobre los indicadores de dinámica laboral. Para esto fueron incorporadas en los cuestionarios de campo dos preguntas referidas a cambios en la cantidad y/o calificación del empleo como resultados de las actividades de innovación declaradas por la empresa. En tal sentido, éste propone tres etapas para la comprobación empírica de las 
hipótesis: en la primera se busca identificar las estrategias de innovación seguidas por las empresas, en la segunda se indaga el efecto de las capacidades en la determinación de las estrategias y en la tercera el impacto de las estrategias y capacidades sobre cambios en el empleo.

El resto del artículo se organiza de la siguiente manera: En la sección I se presentan los principales antecedentes del estudio sobre la relación entre innovación y empleo, así como el marco conceptual y teórico propuesto. En seguida se plantean las hipótesis de trabajo derivadas del marco teórico. La sección II discute las principales características del relevamiento así como una descripción estadística de la muestra obtenida. La sección III presenta los lineamientos metodológicos y también los resultados obtenidos en la comprobación empírica de las hipótesis de trabajo. Finalmente, en la última sección se plantean las principales conclusiones.

\section{ANTECEDENTES Y MARCO TEÓRICO DE REFERENCIA}

Los trabajos que indagan sobre la relación entre innovación y empleo pueden categorizarse en función de dos dimensiones de análisis (Pianta, 2006). La primera dimensión distingue los enfoques de equilibrio, principalmente asociados con la teoría neoclásica walrasiana como labor economics o la nueva teoría del crecimiento endógeno, respecto de los de desequilibrio, que aglutinan las aportaciones de la escuela evolucionista, del estructuralismo o de la teoría de la regulación, entre otros.

La segunda dimensión hace referencia al nivel de agregación en el que se analiza la relación entre innovación y empleo: nivel de empresa, de industria, o bien, macroeconómico. La referencia al nivel de agregación responde a una preocupación sobre los efectos contrapuestos en el empleo causados por la innovación o el cambio tecnológico. De tal forma, lo que puede ser cierto en el nivel de empresa puede no serlo en un nivel agregado, ya que la creación de empleo puede verse compensada por efectos de destrucción de puestos. Estos efectos compensatorios pueden ser tanto interindustriales como intraindustiales, según estén vinculados con el surgimiento de nuevos sectores y nuevos productos, o si se relacionan con el cambio en la estructura de competencia al interior de un sector (market share). Por ejemplo, el incremento de competitividad de una empresa $\mathrm{y}$, por ende, del empleo, puede verse reflejado en el cierre de competidores.

Esta inquietud por los efectos de compensación en la generación de empleo, como resultado de introducir innovaciones en la actividad productiva, tiene 
una larga tradición que se remonta a autores de la economía clásica. En un nivel elevado de agregación coexisten procesos de destrucción de empleo, generalmente asociados con el ahorro de mano de obra, inducidos por la innovación, con múltiples canales de creación de empleo. De forma similar, es de esperar que introducir innovaciones demande perfiles de mayor calificación. Sin embargo, puede argumentarse que incorporar maquinaria o cambios organizacionales permitió a la empresa prescindir de los trabajadores de mayor calificación e incorporar en los procesos y maquinarias el savoir-faire que era el principal dominio del trabajador (Coriat, 1992). Por tanto, los efectos netos positivos o negativos en términos de cantidad de trabajadores, así como la composición del empleo en términos de calificaciones, permanecen a priori indeterminados.

A pesar de que la relación entre innovación y empleo es de larga data, durante las décadas de los ochenta y noventa parece haber resurgido en la bibliografía un fuerte interés por las vinculaciones entre el cambio tecnológico y la dinámica del empleo. La principal motivación que subyace a esta nueva ola de trabajos teóricos y empíricos ${ }^{1}$ estuvo orientada a explicar dos hechos aceptados: el incremento de la desigualdad salarial en los Estados Unidos desde fines de la década de los setenta y el aumento del desempleo europeo durante la década de los noventa.

Por ejemplo, Acemoglu (2002) explica el incremento de la desigualdad en los Estados Unidos a partir del sesgo tecnológico en perjuicio de los trabajadores menos calificados (skill-biased technology), lo cual a su vez le permite explicar el incremento en la disparidad salarial. Por su parte, Berman, Bound y Griliches (1994) muestran cómo el cambio tecnológico ahorrador de mano de obra constituye la mejor explicación del cambio en la demanda relativa de calificaciones, más que el mayor crecimiento de sectores intensivos en conocimiento. Por último, otras aportaciones han centrado su análisis en el impacto que diversos tipos de innovación tendrían en el mercado laboral, buscando hallar sustento a la idea de desempleo tecnológico que podría explicar la situación del mercado laboral europeo.

El estudio de los efectos compensatorios netos, atractivo desde el punto de vista de la incorporación de múltiples canales de trasmisión como parte del análisis, puede volverse una dificultad significativa a la hora de cuantificar y

\footnotetext{
${ }^{1}$ Chennels y Van Reenen (1999) identifican más de 70 trabajos empíricos, en los que se demuestra la existencia de un fuerte impacto de la tecnología en las demandas relativas de calificaciones y en la estructura salarial.
} 
establecer relaciones causales individuales. Esta debilidad motiva el estudio a menores niveles de agregación. Los estudios a nivel industria permiten identificar dinámicas sectoriales específicas y captar de manera más definida las características del proceso de innovación en el marco de un régimen tecnológico particular (Malerba y Orsegino, 1997). Por ejemplo, la definición poco precisa de "innovación" que suele emplearse habitualmente (como el concepto de productividad total de los factores, utilizado en la economía neoclásica) puede ser reemplazada por definiciones acotadas a un contexto tecnológico e institucional dado.

Al mismo tiempo, el análisis a nivel de la industria, al permitir dar cuenta de las diferentes respuestas del empleo frente a la innovación, puede contribuir a entender la dinámica agregada con base en el análisis de la estructura productiva del país. Entre los hechos aceptados en el nivel de industria o sector, la biblio $^{\circ}$ grafía ha detectado que diferentes sectores tienen dinámicas específicas de innovación y que el impacto sobre el empleo puede variar en función de esto (Pianta, 2006). En consecuencia, el perfil de especialización productiva de un país puede afectar el funcionamiento de los mecanismos de compensación.

La bibliografía centrada en el estudio a nivel de empresa, entre la cual se enmarca el presente artículo, si bien no permite captar los efectos compensatorios, tanto intraindustriales como interindustriales, permite ver el efecto directo sobre el empleo de diferentes estrategias de innovación, difícilmente perceptible a niveles más agregados de análisis. Así, por ejemplo, bajo un enfoque de equilibrio, esto se traduce en una estimación directa de la función de demanda de empleo, incorporando esfuerzos de innovación como un insumo en la función de producción de la empresa (Piva y Viviarelli, 2005). Los enfoques de desequilibrio, por su parte, apuntan al estudio cualitativo de diversas estrategias de innovación e involucran crecientemente las capacidades de las empresas, los niveles de vinculaciones entre ellas, la organización de la producción, etcétera. En este esquema, un grupo de trabajos (Brouwer, Kleinknecht y Reijnen, 1993; Jaumandreu, 2003; Hall, 2008; Peters, 2005; Benavente y Lauterbach, 2008, entre otros), generalmente basados en diversas versiones de las encuestas tecnológicas, buscó comprender el diferente impacto de las innovaciones de producto, proceso, comercialización u organización sobre la cantidad y calidad del empleo.

En este sentido, las capacidades de las empresas, las actividades de innovación y los cambios en la cantidad y calidad del trabajo son relaciones ampliamente propuestas en diversos trabajos empíricos (Stoneman, 1995; Van Reenen, 1997; Pianta, 2006; Vivarelli, Evangelista y Pianta; 1996; Evangelista y Savona, 2001; Edquist, 2000; Petit, 1998; Bower et al., 2005; Tether, 2000; Narula, 2001, 
entre otros). Con la excepción de Benavente y Lauterbach (2006) y Novick et al. (2009), la mayoría de los trabajos que analizan la relación entre innovación y empleo se han efectuado en los países desarrollados, por el cual el presente trabajo adquiere relevancia para interpretar estas relaciones en empresas industriales en países en desarrollo.

Entre los hechos aceptados identificados a nivel de empresa se destaca que el tipo de innovación es relevante para entender los efectos en el empleo. Como propone Pianta (2006), la innovación de producto tendría un efecto positivo en la creación de empleo mientras que los impactos de las innovaciones de proceso suelen ser negativos. Asimismo, las innovaciones organizacionales relacionadas con el cambio tecnológico (por ejemplo, difusión de las TIC) afectan no sólo la cantidad sino también la calificación demandada. El cuadro 1 sintetiza los enfoques planteados para cada nivel de análisis.

\section{Cuadro 1. Distintos enfoques en innovación y empleo*}

\begin{tabular}{|c|c|c|}
\hline $\begin{array}{l}\text { Enfoque general } \\
\text { Nivel de análisis }\end{array}$ & Equilibrio & Desequilibrio \\
\hline $\begin{array}{l}\text { Macro: Cambios en el volumen } \\
\text { de empleo debido a innovacio- } \\
\text { nes. Naturaleza del desempleo } \\
\text { (friccional o tecnológico). }\end{array}$ & $\begin{array}{l}\text { Mecanismos de compensa- } \\
\text { ción (clásicos: Ricardo y Marx). } \\
\text { Cambios en el total de em- } \\
\text { pleo son irrelevantes debida } \\
\text { a los mecanismos de compen- } \\
\text { sación. Foco en innovaciones } \\
\text { de proceso (labor economics, } \\
\text { crecimiento y nuevas teorías } \\
\text { de crecimiento). }\end{array}$ & $\begin{array}{l}\text { Los mecanismos de compensación } \\
\text { pueden no funcionar, llevando a la } \\
\text { existencia de desempleo tecnológi- } \\
\text { co. Consideran ondas largas en el } \\
\text { cambio tecnológico y mismatching } \\
\text { (evolucionistas y neoschumpeteria- } \\
\text { nos). Las cuestiones institucionales } \\
\text { son relevantes (regulacionistas). }\end{array}$ \\
\hline $\begin{array}{l}\text { Industria: Especificidades } \\
\text { sectoriales de la innovación. }\end{array}$ & $\begin{array}{l}\text { Análisis de equilibrio parcial. } \\
\text { Demografía de trabajo. Flexi- } \\
\text { bilidad del mercado de traba- } \\
\text { jo (labor economics). }\end{array}$ & $\begin{array}{l}\text { La innovación acarrea desequilibrios. } \\
\text { Procesos de destrucción creativa } \\
\text { (evolucionistas y neoschumpeteria- } \\
\text { nos). Las cuestiones institucionales } \\
\text { son relevantes (regulacionistas). }\end{array}$ \\
\hline $\begin{array}{l}\text { Empresa: Cantidad de puestos } \\
\text { de trabajo creados/perdidos de- } \\
\text { bido a la innovación. Impacto } \\
\text { de los diferentes tipos de inno- } \\
\text { vación sobre el empleo. Cam- } \\
\text { bios en la calidad del empleo y } \\
\text { en la calificación de los trabaja- } \\
\text { dores. }\end{array}$ & $\begin{array}{l}\text { Objetivo: estimar la demada } \\
\text { de empleo de una empresa } \\
\text { representativa aumentada } \\
\text { por actividades de innova- } \\
\text { ción. Foco en las innovaciones } \\
\text { de proceso (labor economics). }\end{array}$ & $\begin{array}{l}\text { Analizan la innovación y la difusión } \\
\text { de innovaciones. Consideran hetero- } \\
\text { geneidad de empresas según sus ca- } \\
\text { pacidades y trayectorias. Las diferen- } \\
\text { cias en materia de oportunidad, } \\
\text { variedad y acumulatividad tecnológi- } \\
\text { ca llevan a diferentes conductas inno- } \\
\text { vativas y diferencias en su impacto } \\
\text { sobre el empleo. Diferentes tipos de } \\
\text { innovación (producto, proceso) tie- } \\
\text { nen diferente impacto sobre el em- } \\
\text { pleo (evolucionistas). }\end{array}$ \\
\hline
\end{tabular}

* El enfoque específico está indicado entre paréntesis. Fuente: Elaboración propia, basada en Pianta (2006). 
Los antecedentes presentados muestran que el interés de la bibliografía a nivel de empresa se ha centrado en identificar factores con efectos positivos sobre el empleo o la composición en términos de calificaciones. Sin embargo, desde una perspectiva evolucionista, las actividades de innovación de las firmas no se generan en forma desarticulada sino en el marco de estrategias que combinan esfuerzos y resultados diversos.

Estas estrategias estarían limitadas por las capacidades endógenas de la empresa y determinadas por la experiencia acumulada y el conocimiento adquirido, así como por las oportunidades existentes en la rama productiva en la que operan. Así, es necesario comprender la formación de las estrategias de innovación como el resultado emergente de un proceso complejo sujeto no sólo a las decisiones del empresario, sino también a las capacidades de absorción y de conectividad acumuladas a lo largo de su sendero de aprendizaje en el que intervienen tanto factores internos como externos a la empresa.

Este artículo se inscribe en el enfoque evolucionista de la economía de la innovación, ampliado por el marco general de sistemas complejos (Antonelli, 2008). En este sentido, el artículo comparte la hipótesis de la existencia de una dinámica no lineal de los procesos de aprendizaje de las empresas y la innovación como una propiedad emergente del sistema.

El enfoque de capacidades presupone la existencia de una amplia heterogeneidad micro definida en términos de capacidad de absorción y de conectividad. La capacidad de absorción está definida como la capacidad de la empresa o firma para reconocer nueva información externa, asimilarla y aplicarla (Cohen y Levinthal, 1989) y depende de factores tales como la organización del trabajo, las actividades de capacitación, la existencia de equipos de ID y la gestión de la calidad de la organización. Por su parte, la capacidad de conectividad de las empresas refiere a la potencialidad de éstas de establecer relaciones y generar interacciones con el objetivo de incrementar su base de conocimiento (P. Norman, 2002; P. A. Cullen, 2000; Grandori y Soda, 1995, Powell y Grodal, 2005). Esta capacidad es función de la calidad de las vinculaciones que establece una organización con otras empresas u otras instituciones (universidades, centros tecnológicos, etcétera). Así, las capacidades de absorción y conectividad se refuerzan mutuamente.

Las organizaciones con capacidad de absorción elevada tienden a ser más abiertas y sostienen una mayor densidad en sus relaciones. Al mismo tiempo, la densidad de relaciones (la capacidad de conectividad) ayuda a desarrollar 
una mayor capacidad de absorción ya que la organización está expuesta a flujos significativos de conocimiento de los que aprende y desarrolla nuevas capacidades. En esta dirección, una de las principales hipótesis del trabajo es que diferentes niveles en ambas capacidades mejoran los procesos de aprendizaje de la empresa y las posibilidades de apropiación de las rentas derivadas de la innovación.

En este sentido, se considera que las capacidades no tienen sólo un efecto indirecto sobre la generación de empleo, a partir de su impacto sobre las estrategias de innovación, sino también directo, en la medida en que las empresas de mayores capacidades podrán mejorar su posición competitiva y expandir su tamaño. Paralelamente, también se plantea la necesidad de tener en cuenta los efectos sectoriales. Esto surge de asumir la innovación como un fenómeno no circunscrito a la esfera de la empresa sino como un proceso que se desarrolla a partir de las vinculaciones entre agentes, con carácter sistémico.

En este marco, a continuación se plantean las hipótesis de trabajo que probaremos en las secciones empíricas del artículo.

Hipótesis 1. Existe una relación de causalidad entre el nivel alcanzado por las capacidades de las empresas (absorción y conectividad) y el tipo de estrategia de innovación seguida por éstas. En particular, mayores capacidades adquiridas se corresponden con estrategias más refinadas de innovación.

Hipótesis 2. Los cambios en la cantidad de trabajadores se encuentran causalmente asociados con $i$ ) el tipo de estrategia de innovación, y ii) las capacidades de absorción acumuladas.

Hipótesis 3. Los cambios en la calificación de los trabajadores se encuentra causalmente asociada con $i$ ) el tipo de estrategia de innovación; ii) las capacidades de absorción acumuladas, y iii) los incrementos en la cantidad de trabajadores derivados de la innovación.

Hipótesis 4. Existen efectos sectoriales en los cambios en el empleo (cantidad y calificación) debido a la innovación.

En la figura 1 se esquematizan las relaciones entre las variables planteadas en la hipótesis de trabajo 4. 
Figura 1. Esquema de relaciones

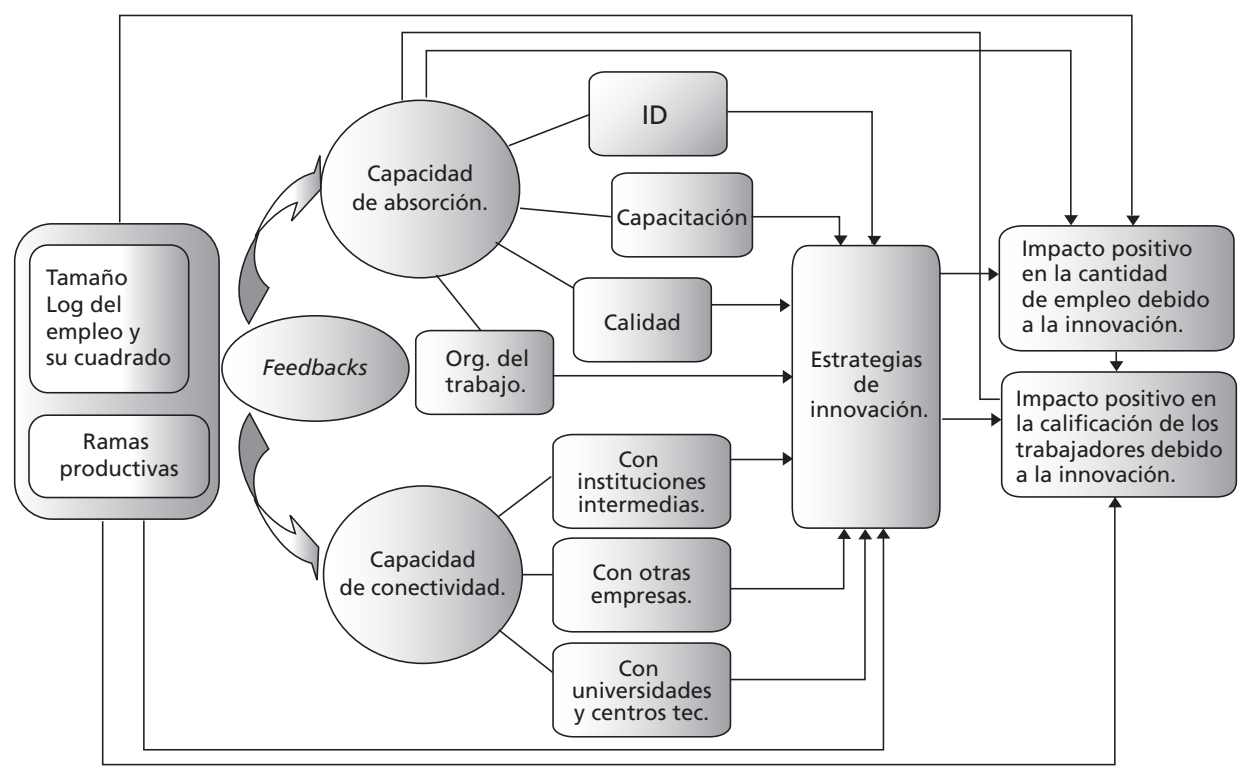

Fuente: Elaboración propia.

\section{DESCRIPCIÓN DE LA MUESTRA}

El ejercicio empírico propuesto para probar las hipótesis de trabajo fue realizado a partir de una encuesta llevada a cabo en 403 empresas industriales pertenecientes a siete ramas productivas, seis de ellas definidas sectorialmente y una geográficamente. Las seis ramas sectoriales corresponden a maquinaria agrícola $(14.1 \%$ del total de la muestra); textil, confecciones intensivas en diseño (10.3\%); embarcaciones $(4.8 \%)$; servicios petroleros $(6.8 \%)$; autopartes $(21.9 \%)$ y siderurgia (19.6\%). El grupo de firmas localizadas en Morón (el 22.6\% restante) involucra a un conjunto de empresas industriales que pertenecen a diversos sectores, entre los que predomina la rama metalmecánica.

Los trabajos de campo se realizaron entre 2005 y 2008. La encuesta procuraba recabar información sobre el grado de desarrollo de capacidades y conducta innovadora de empresas industriales pertenecientes a ramas productivas. El formulario aplicado consideró diferentes ámbitos relevantes para evaluar capacidades e innovación, entre ellas: gestión de la calidad, organización del trabajo, vinculaciones, actividades y resultados de innovación. En lo referente a la 
evolución del empleo, la encuesta no sólo reveló el nivel y evolución reciente de la cantidad y composición (en términos de calificaciones) del empleo, sino que también consideraba una pregunta acerca de la evolución del empleo como respuesta a las actividades de innovación realizadas. ${ }^{2}$

La decisión de operar sobre ramas y no sectores responde al interés de superar la visión tradicional de segmentación del aparato productivo que no siempre considera las interacciones de conocimiento que pueden darse entre empresas que no pertenecen a un mismo sector, pero sí comparten, por ejemplo, la pertenencia a una misma cadena de valor. De tal forma, las ramas siderúrgica y automotriz fueron definidas sobre la base de la red de proveedores (y clientes, en el caso de la siderurgia) de las usinas integradas y las terminales automotrices. De forma similar, se definió la rama de servicios petroleros como aquellas empresas de servicios que proliferaron a partir de la privatización de YPF, la empresa pública de exploración, explotación y destilación de petróleo, subcontratista de la actual Repsol-YPF y de otras empresas petroleras. Las ramas de embarcaciones (que aglutina a las empresas que producen fundamentalmente embarcaciones deportivas) y de confecciones (que considera a las empresas de confecciones con contenido de diseño) no sólo tuvieron un recorte sectorial sino también geográfico, asumiendo que el territorio en estos casos representa una variable importante para entender los flujos de conocimiento. Por último, el grupo de empresas manufactureras de Morón es el único caso de determinación geográfica, aunque cabe mencionar que la presencia de empresas metalmecánicas es predominante. ${ }^{3}$

En términos de tamaño, las empresas de la muestra presentan cierta heterogeneidad. Un 36\% de las empresas tiene menos de 20 empleados, lo que representa $4 \%$ del total de los 35000 empleados cubiertos por la muestra. En el extremo opuesto, las empresas de más de 100 ocupados cubren $21 \%$ del universo estudiado, con una participación en el empleo total de 69\% (sólo 3\% de las firmas de la muestra tiene más de 500 ocupados). El mayor predominio de empresas

\footnotetext{
${ }^{2}$ En el anexo metodológico se incluye una versión resumida del formulario aplicado.

${ }^{3}$ En los casos en los que una empresa pudiera pertenecer a más de una rama, se establecieron diferentes criterios para decidir su categorización; por ejemplo, se priorizó el sector respecto a la localización. Prácticamente en todas las ramas productivas analizadas, las muestras representan una alta proporción del universo. Así, para el primer y segundo anillo de proveedores de la industria automotriz, la muestra representa cerca de $35 \%$, mientras que en proveedores y clientes de la industria siderúrgica (rama siderúrgica) este porcentaje es cercano a $30 \%$, y en los casos de maquinaria agrícola y empresas manufactureras de Morón, ronda el 25\%. Por lo contrario, este promedio es menor en las ramas de embarcaciones $(10 \%)$ y en confecciones $(20 \%)$. Para mayor detalle sobre los trabajos de campo realizados véase Erbes, et al. (2009).
} 
de menor tamaño en la muestra es congruente con la composición por tamaño de las ramas analizadas, en especial cuando abordamos los casos de las ramas textilconfecciones, embarcaciones y las empresas manufactureras de Morón, donde predominan las empresas pequeñas y medianas (véase cuadros A1 y A2 del anexo estadístico).

A partir del cuestionario, se identificó un conjunto de variables que permiten una caracterización rápida de la muestra. La presencia de capital extranjero, la pertenencia a grupos empresarios y la participación en mercados externos suelen utilizarse frecuentemente como variables de control. La certificación de calidad también se consideró, dada la centralidad que tienen los procesos de aprendizaje y de innovación en este artículo. En lo que respecta al origen del capital, 356 de las 403 empresas son de capital nacional (88\%), en tanto que las 47 restantes son el resultado de inversiones extranjeras directas con distinta proporción en el capital accionario. Las empresas de capital extranjero tienen mayor presencia en la rama automotriz. El coeficiente de exportación de la muestra es de $18 \%$ en promedio, con diferencias significativas entre las ramas estudiadas. En lo que respecta a la presencia de normas de calidad, cerca de $50 \%$ de las empresas cuentan con certificación de aseguramiento de la calidad. Las empresas correspondientes a las ramas automotriz, siderúrgica y de servicios petroleros son las que con mayor frecuencia han certificado calidad (véase cuadro A2 del anexo estadístico).

El nivel de desarrollo de las capacidades de absorción y conectividad de las empresas de la muestra fue estimado a partir de un conjunto de variables. En lo que respecta al indicador de capacidad de absorción, se consideraron cuatro dimensiones: $i$ ) la gestión de la calidad (control de procesos, cultura hacia la calidad y utilización de herramientas de mejora continua); ii) la capacitación (actividades y existencia de estructura de capacitación); iii) la organización del trabajo (rotación y equipos de trabajo vs. puestos individuales), y $i v$ ) la presencia y tipo (formal o informal) de grupos de ID. ${ }^{4}$

El enfoque multifacético utilizado para evaluar la capacidad de absorción responde a un desencuentro entre la muy rica definición teórica de esta capacidad y los intentos generalmente simplistas de estimarla, entre los cuales generalmente sólo es considerada la ID (Van den Bosch, et. al. 1999; Zahra y George, 2002; Cullen, 2000, David et al., 1996; Coriat y Weinstein, 2002; Roitter

\footnotetext{
${ }^{4}$ Para mayor detalle sobre la construcción de los indicadores de gestión de la calidad, véase el apéndice metodológico.
} 
et al. 2006, y Erbes, Robert y Yoguel, 2010). Por su parte, para la estimación de la capacidad de conectividad se consideraron tres diferentes tipos de patrones relacionados con el tipo de agente con el que se establecen las vinculaciones de las empresas: $i$ ) otras empresas, como clientes, proveedores y competidores; ii) instituciones intermedias, compuestas por cámaras empresariales o consultores, y iii) instituciones académicas y de investigación, como universidades y centros de ciencia y tecnología (Roiter et al., 1997, Yoguel et al., 2009). Con el objetivo de evaluar la calidad de las vinculaciones fueron contemplados los objetivos de éstas, asumiendo que las vinculaciones con objetivos múltiples serían más complejas y, por lo tanto, de mejor calidad (véase anexo metodológico).

En términos generales, el nivel de desarrollo de las capacidades de absorción de las empresas de la muestra es reducido, con importantes diferencias entre las ramas estudiadas. Por ejemplo, la presencia de un grupo formal de ID se observa en $26 \%$ de las empresas de la muestra, porcentaje que asciende a 51 y $58 \%$ en las ramas de maquinaria agrícola y automotriz, respectivamente, y cae por debajo de $10 \%$ en las empresas de la rama de embarcaciones. En lo que respecta a la capacidad de conectividad, puede observarse que entre 30 y $40 \%$ de las empresas de la muestra han mantenido vínculos con al menos algún tipo de agente, con cierta heterogeneidad entre las ramas. Sin embargo, la calidad de la vinculación predominante es la baja. Sólo entre 4 y $7 \%$ de las empresas de la muestra presentaron vinculaciones de alta calidad (véase cuadro A5 del anexo estadístico).

Para el análisis de la conducta innovativa de las empresas, los cuestionarios utilizados incorporaron un amplio conjunto de preguntas en la búsqueda de información sobre esfuerzos y resultados, así como otras acerca de las características más estructurales, como sus capacidades técnicas u organizacionales. Uno de los indicadores más habituales se construye sobre la base de los resultados de innovación, medidos en cuatro ámbitos: proceso, producto, organización y comercialización. El 76\% de las empresas afirmaron haber obtenido algún resultado de innovación de producto y $69 \%$ en innovaciones de proceso. En lo referente a resultados en los ámbitos de organización y comercialización, 45 y $32 \%$ ha respondido, respectivamente, ${ }^{5}$ de manera afirmativa (véase cuadro A4 de anexo estadístico).

Esa primera aproximación, que parecería presentar una visión algo sobreestimada de las actividades innovativas de las empresas, resulta reafirmada al

${ }^{5}$ En todos los casos, las empresas debían aclarar en qué consistía el resultado pertinente, lo que se utilizaba para validar las respuestas. 
analizar las cuatro respuestas para conocer el número total de resultados de innovación o, como suele denominarse, el grado de equilibrio de las innovaciones ${ }^{6}$ (véase cuadro A4 de anexo estadístico). Así, 20\% de las empresas entrevistadas afirmaron haber innovado en las cuatro categorías. En el extremo opuesto, $85 \%$ reconoció haber alcanzado resultados en por lo menos una de las cuatro áreas. Esta cifra resulta llamativa si es comparada con resultados de otros países. Por ejemplo, de acuerdo con la encuesta de innovación europea, durante 2004 el porcentaje de empresas innovadoras en Alemania resultó ser de 65\%; en el caso de Francia, de 41\%; en Italia, 36\%, y en España, 33 por ciento. ${ }^{7}$

Una interrogante adicional en relación con estos indicadores surge de los cruces presentados en el cuadro 2. Cada recuadro asocia un tipo particular de innovación con el hecho de que la nómina laboral haya crecido durante el periodo 2001-2006. ${ }^{8}$ En virtud de lo planteado en el marco teórico, se esperaría la existencia de vinculación entre estas variables y, sin embargo, en todos los casos las variables muestran indicios de independencia a partir de las pruebas chi cuadrada de correlación de Pearson aplicados.

Los problemas planteados muestran las dificultades que implica el uso de indicadores basados en resultados de innovación para evaluar la dinámica del empleo, en términos de calificación y cantidad. Estos problemas pueden estar asociados con la interpretación del entrevistado sobre qué es lo que efectivamente se entiende por "resultado de innovación en procesos, productos, etcétera". 9

\footnotetext{
${ }^{6}$ A partir del indicador "número de innovaciones" se da cuenta del grado de equilibrio en los resultados obtenidos. Así, en la medida en que los resultados de innovaciones involucren diferentes ámbitos, se puede hablar de resultados más equilibrados. Es de esperar que la introducción de nuevos productos involucre cambios en los procesos productivos y en la comercialización. Incluso pueden verse al mismo tiempo transformaciones organizacionales acordes. En este caso, los cuatro tipos de innovación estarían presentes y los resultados, por tanto, estarían equilibrados. Si se obtienen resultados en un solo campo, es posible que la innovación sea de poca importancia ya que no obliga a realizar cambios en los restantes. En este caso se hablaría de resultados desequilibrados, tales como cambios organizacionales aislados.

${ }^{7}$ De acuerdo con la cuarta encuesta comunitaria de innovación (CIS-4), existen serias dificultades en la comparación asociadas con cuestiones tales como diferencias en los formularios, en las estructuras productivas o en las formas de interpretación de los formularios. No obstante, consideramos que la comparación es válida si el objetivo es señalar los problemas de medición en el caso del cuestionario aplicado a las ramas productivas argentinas.

${ }^{8}$ Para los casos donde no existía la información correspondiente a 2001, se utilizó el dato correspondiente a 2003.

${ }^{9}$ La pregunta referida a los resultados de innovación fue formulada de la siguiente manera en el cuestionario: “¿Podría señalar si realizó innovaciones en productos/servicios, procesos, comercialización y organización del trabajo?”. Esto supone que el encuestado maneja una definición de
} 


\section{Cuadro 2. Correlación entre resultados de innovación y variación en el nivel de empleo}

\begin{tabular}{|c|c|c|c|c|}
\hline \multicolumn{2}{|c|}{$\begin{array}{l}\text { ¿Ha desarrollado } \\
\text { innovaciones... }\end{array}$} & \multicolumn{2}{|c|}{$\begin{array}{l}\text { ¿Aumentó el número de emplea- } \\
\text { dos entre } 2001 \text { y } 2006 ? \\
\text { No }\end{array}$} & Total \\
\hline \multirow{3}{*}{$\begin{array}{r}\ldots \text { de } \\
\text { producto? }\end{array}$} & Sí & 45 & 187 & 232 \\
\hline & No & 21 & 71 & 94 \\
\hline & Total & 100 & 100 & 100 \\
\hline \multicolumn{5}{|c|}{ Pearson $\chi^{2}=0.359 p=54.9 \%$} \\
\hline \multirow{3}{*}{$\begin{array}{r}\ldots \text { de } \\
\text { proceso? }\end{array}$} & Sí & 42 & 126 & 168 \\
\hline & No & 24 & 64 & 88 \\
\hline & Total & 100 & 100 & 100 \\
\hline \multicolumn{5}{|c|}{ Pearson $\chi^{2}=0.391 p=53.2 \%$} \\
\hline \multirow{3}{*}{$\begin{array}{r}\text {...organiza- } \\
\text { cionales? }\end{array}$} & Sí & 30 & 117 & 147 \\
\hline & No & 36 & 143 & 179 \\
\hline & Total & 100 & 100 & 100 \\
\hline \multicolumn{5}{|c|}{ Pearson $\chi^{2}=0.044 p=94.7 \%$} \\
\hline \multirow{3}{*}{$\begin{array}{r}\text {... de } \\
\text { comercia- } \\
\text { lización? }\end{array}$} & Sí & 17 & 89 & 106 \\
\hline & No & 49 & 171 & 220 \\
\hline & Total & 100 & 100 & 100 \\
\hline \multicolumn{5}{|c|}{ Pearson $\chi^{2}=1.723 p=18.9 \%$} \\
\hline
\end{tabular}

Fuente: Elaboración propia con base en encuestas a ramas productivas argentinas.

Estas debilidades incentivan la búsqueda de mejores indicadores para aproximarse a la conducta innovativa de las empresas. En este contexto, en la

innovación cercana a la utilizada en economía de la innovación. Por ejemplo, es posible que el encuestado considere por innovación lo que en la bibliografía especializada se entiende como innovaciones radicales, es decir, productos o procesos completamente novedosos. En estos casos es muy probable que el encuestado tienda a subestimar sus respuestas. Por ello, el cuestionario agregaba el siguiente comentario: "Como 'innovación' consideramos mejoras significativas en el plano tecnológico y de la gestión de la empresa, las cuales pueden darse tanto por desarrollo propio como por adopción de tecnologías desarrolladas por otras empresas e instituciones, o por adaptaciones de las mismas". Este comentario, si bien busca resolver los problemas de subestimación por la no consideración de innovaciones para la empresa pero no para el mercado, induce a su vez nuevas fuentes de subjetividad en las respuestas, especialmente debidas al término de "mejoras significativas". Este hecho, sumado a que la innovación es crecientemente considerada como una práctica altamente deseable y fuertemente cargada de connotaciones positivas, pudo provocar en las respuestas una fuerte sobreestimación de los resultados, que es lo que observamos al comparar con otras fuentes de información. 
próxima sección describiremos la metodología aplicada para construir estrategias de innovación a partir de las respuestas de las empresas sobre los esfuerzos de innovación realizados. Al respecto, cabe explicitar dos cuestiones: en primer lugar, y desde el contexto de relevamiento de la información, entendemos que las respuestas sobre esfuerzos de innovación involucran un menor contenido de subjetividad que las respuestas sobre resultados. En general, es de esperar menor discrecionalidad al responder afirmativa o negativamente sobre la compra de bien de capital, la adquisición de licencias, la incorporación o desarrollo de software, etcétera. ${ }^{10}$

\section{COMPROBACIÓN DE HIPÓTESIS EN TRES ETAPAS}

\section{Primera etapa: Definición de las estrategias de innovación}

El primer paso del ejercicio econométrico fue la determinación y caracterización de las estrategias de innovación seguidas por las empresas para dar cuenta de la conducta innovativa de una manera compleja y no como la simple adición de esfuerzos aislados.

Para su construcción, fue considerada la pregunta referida a esfuerzos de innovación, de la cual se extrajeron ocho variables dicotómicas que reflejan la realización de esfuerzos específicos: $i$ ) adquisición de bienes de capital, ${ }^{11}$ ii) adquisición de licencias; iii) adquisición de software; iv) desarrollo de software; v) diseño y desarrollo de productos; vi) diseño y desarrollo de canales de comercialización, vii) desarrollo de procesos, y viii) búsqueda e investigación de oportunidades científicas.

Las ocho variables luego fueron agrupadas combinando el método de análisis factorial y el de cluster. A partir del análisis factorial es posible reducir la dimensionalidad del fenómeno estudiado, al reducir el número de variables observables a un conjunto menor de variables no observables denominadas factores. A partir de allí, las observaciones son agrupadas en una cantidad predeterminada de clusters buscando minimizar la variabilidad entre los componentes de cada grupo y maximizar la variabilidad entre los grupos. Esta metodología permitió agrupar los esfuerzos individuales de innovación en estrategias de distinto tipo, dando cuenta así de un fenómeno complejo que muchas veces escapa a la

\footnotetext{
${ }^{10}$ La pregunta en este caso se refiere a si la empresa realiza o no una serie de actividades referidas a esfuerzos de innovación (véase anexo metodológico).

${ }^{11}$ Descartando aquellos que implicaran la simple reposición de maquinaria.
} 
categorización convencional de los esfuerzos de innovación tomados de manera aislada o de la construcción de indicadores ordinales que indiquen el grado de la capacidad innovativa de las empresas.

La principal ventaja de esta metodología frente a los indicadores ordinales surge del no requerimiento de categorías predefinidas que pueden incorporar un componente $a d-h o c$ al análisis. Mediante la utilización del análisis factorial, las estrategias de innovación se autodeterminan a partir de los comportamientos efectivamente observados en materia de esfuerzos de innovación. Esto implica otras ventajas adicionales que adquieren especial relevancia dado el esquema teórico propuesto. En primer lugar, esta metodología no permite determinar $a$ priori la superioridad de una estrategia en relación con las restantes. Por tanto, puede sostenerse el supuesto teórico de que cada estrategia es esencialmente diferente y está estrechamente vinculada con las características estructurales de la empresa en cuestión - como el tamaño o rama en la que opera- y con las capacidades de la misma. En segundo lugar, el esquema analítico-metodológico propuesto no da lugar a la definición teórica de una estrategia óptima o de benchmark contra la cual establecer las comparaciones. Si este fuera el caso, el objetivo sería mostrar qué variables acercan o alejan a la estrategia efectivamente adoptada de este ideal.

En nuestro caso suponemos la diversidad como un componente esencial de los sistemas en los que los agentes compiten y, por tanto, no creemos que sea posible establecer un ideal conveniente para que todas las empresas converjan. En oposición, cada empresa tendrá una estrategia de innovación específica, que bajo nuestras hipótesis de trabajo 1 y 2, dependerán de su sendero evolutivo y por tanto estarán impregnadas de las características que afectan las competencias técnicas y organizacionales de la misma. Esta dimensión de análisis se complementa con el modelo de regresión multinomial propuesto para la comprobación de las primeras hipótesis del trabajo.

Una aclaración que merece hacerse es que si bien no es posible establecer a priori un orden completo entre las estrategias obtenidas mediante el análisis factorial, esto no implica que éstas no puedan ser caracterizadas incluso valorativamente. ${ }^{12}$ De modo similar no deben derivarse de lo expuesto aquí reco-

\footnotetext{
${ }^{12}$ De hecho vamos a identificar entre los grupos obtenidos estrategias más débiles que otras. Esto no es una contradicción con lo anteriormente expuesto; no es posible determinar claramente cuál es la mejor estrategia, ya que esto depende directamente del agente que adopta la misma. Una estrategia muy buena para una empresa que opera en la rama siderúrgica puede incluir fuertemente el equipamiento de capital sobre los esfuerzos desincorporados; un caso opuesto podría presentarse
} 
mendaciones de política del tipo liberal, bajo la presunción de que cada empresa maximiza su estrategia dada una restricción tecnológica o de mercado. Por lo contrario, como mostraremos en las secciones siguientes, de este enfoque se desprenden recomendaciones de política industrial activa.

En la medida en que las estrategias de innovación más débiles estén asociadas con sectores poco dinámicos y con empresas con bajas capacidades, entonces las recomendaciones apuntan: $i$ ) a fortalecer las capacidades endógenas de las empresas y el enramado productivo en el que operan, y ii) a lograr una mayor complejidad del perfil productivo con mayor participación en sectores dinámicos.

Del análisis propuesto fueron identificados cuatro grupos de empresas. Cada grupo se caracterizó por realizar un conjunto más o menos definido de esfuerzos de innovación. De este modo puede afirmarse que las empresas que componen cada grupo "se parecen” en términos de los esfuerzos de innovación realizados. Por este motivo decimos que cada grupo define una estrategia de innovación que es elegida por las empresas que lo componen, y que cada estrategia está caracterizada por los esfuerzos que se encuentran sobrerrepresentados en el grupo. ${ }^{13}$

Así, fueron definidas cuatro estrategias de innovación: $i$ ) ausencia de articulación de los esfuerzos de innovación realizados (20\%); ii) basada en bienes de capital (40\%); iii) basada en investigación e incorporación de nuevos productos y procesos $(16 \%)$, y $i v$ ) basada en la incorporación de nuevos productos, procesos y software $(25 \%)$.

La primera de ellas está caracterizada por la falta de coordinación entre los esfuerzos de innovación llevados adelante por la empresa. Este grupo se caracteriza por la subrepresentación de cada uno de los esfuerzos de innovación en particular. Esto no implica que las empresas que componen este grupo "no hagan nada", sino que los esfuerzos de innovación son realizados en forma aislada o no muestran un patrón que las identifique. La segunda estrategia, por su parte, está

en empresas del sector de embarcaciones, donde la flexibilidad del proceso productivo obliga a reducir al máximo los costos fijos.

${ }^{13}$ Cabe aclarar dos cuestiones que frecuentemente conducen a confusiones en el análisis de cluster: primero, no todas las empresas de un grupo deben realizar exactamente los mismos esfuerzos, sino que las combinaciones de esfuerzos realizados por las empresas de un mismo grupo son similares entre sí; segundo, la existencia de esfuerzos sobrerrepresentados no implica: $i$ ) ni que todas las empresas del grupo los realicen necesariamente (aunque es de esperar que un alto porcentaje de empresas en el grupo lo hagan), ii) ni que el resto de los esfuerzos no hayan sido realizados en ninguna medida por las empresas del grupo. 
caracterizada por adquirir bienes de capital. La totalidad de las empresas de este grupo adquirieron bienes de capital, lo cual no implica que no hayan realizado otros esfuerzos sino que este es el tipo de esfuerzo que las distingue. Las tercera y cuarta estrategias coinciden en la sobrerrepresentación de los desarrollos de productos y procesos, pero se diferencian en la adquisición de licencias y búsqueda e investigación de oportunidades científicas, esfuerzos sobrerrepresentados entre las empresas que componen el grupo 3, y desarrollo y adquisición de software, que destaca en la estrategia 4 (véase cuadro 3).

Cuadro 3. Definición de las estrategias de innovación

\begin{tabular}{|c|c|c|c|c|c|}
\hline $\begin{array}{l}\text { Esfuerzos de innovación } \\
\text { (Variaciones nominales acti- } \\
\text { vas en el análisis factorial). }\end{array}$ & $\begin{array}{l}\text { 1. Falta de } \\
\text { una } \\
\text { estrategia } \\
\text { clara de } \\
\text { innovación. }\end{array}$ & $\begin{array}{l}\text { 2. Estrategia } \\
\text { basada } \\
\text { en la adqui- } \\
\text { sición de } \\
\text { bienes de } \\
\text { capital. }\end{array}$ & $\begin{array}{l}\text { 3. Estrategia } \\
\text { basada en la } \\
\text { investigación } \\
\text { de nuevos } \\
\text { productos y } \\
\text { procesos. }\end{array}$ & $\begin{array}{l}\text { 4. Estrategia } \\
\text { basada en la } \\
\text { investigación } \\
\text { de nuevos } \\
\text { productos y } \\
\text { procesos e inc. } \\
\text { soft. }\end{array}$ & Total \\
\hline $\begin{array}{l}\text { Adquisición de bienes de } \\
\text { capital. }\end{array}$ & 0 & $100 *$ & 91 & 90 & 76 \\
\hline Adquisición de licencias. & 5 & 14 & $59 *$ & 24 & 22 \\
\hline Adquisición de software. & 3 & 16 & 10 & 77* & 27 \\
\hline Desarrollo de software. & 3 & 2 & 3 & $86^{*}$ & 23 \\
\hline $\begin{array}{l}\text { Diseño y desarrollo de } \\
\text { producto. }\end{array}$ & 24 & 50 & $87^{*}$ & $84 *$ & 59 \\
\hline $\begin{array}{l}\text { Diseño y desarrollo de canales } \\
\text { de comercialización. }\end{array}$ & 12 & 14 & $49 *$ & $74^{*}$ & 34 \\
\hline Desarrollo de procesos & 20 & 34 & $86 *$ & 71* & 48 \\
\hline $\begin{array}{l}\text { Búsqueda e investigación de } \\
\text { oportunidades científicas. }\end{array}$ & 4 & 3 & 98* & 14 & 21 \\
\hline
\end{tabular}

*Variables sobrerrepresentadas en el grupo (prueba Z con significación $p<0.05$ ).

Fuente: Elaboración propia con base en encuestas a ramas productivas argentinas.

Una evaluación posible de estos resultados consiste en caracterizar a las empresas que siguen la estrategia 1 como las que presentan mayores dificultades en sus capacidades innovativas; las que siguen la estrategia 2 como las que muestran esfuerzos limitados a los del tipo incorporado, y las que siguen las del tipo 3 y 4 como las que lograron una mayor articulación entre esfuerzos incorporados y desincorporados (nótese que alrededor de $90 \%$ de las empresas de ambos grupos adquirió bienes de capital). A priori es difícil decidir si la estrategia 3 es superior a la 4 o viceversa. La incorporación de software, presente en la estrategia 4, da 
muestras de posibles cambios organizacionales, mientras que las actividades de investigación y exploración de oportunidades científicas podrían estar asociadas con altas capacidades endógenas de la organización y a su vez con la pertenencia de sectores con elevadas oportunidades de acuerdo con el concepto de régimen tecnológico de Malerba y Orsenigo (1993).

El cuadro 4 presenta la interrelación entre la rama productiva de pertenencia y los esfuerzos de innovación, lo cual arroja resultados interesantes que permiten una mejor caracterización de las estrategias.

Cuadro 4. Estrategias de innovación según ramas productivas

\begin{tabular}{|c|c|c|c|c|c|}
\hline & $\begin{array}{l}\text { 1. Falta de una } \\
\text { estrategia clara } \\
\text { de innovación. }\end{array}$ & $\begin{array}{l}\text { 2. Estrategia basada } \\
\text { en la adquisición } \\
\text { de bienes de } \\
\text { capital. }\end{array}$ & $\begin{array}{l}\text { 3. Estrategia basada } \\
\text { en la investiga- } \\
\text { ción de nuevos } \\
\text { productos y } \\
\text { procesos. }\end{array}$ & $\begin{array}{l}\text { 4. Estrategia basada en } \\
\text { la investigación de } \\
\text { nuevos productos y } \\
\text { procesos e inc. soft. }\end{array}$ & Total \\
\hline Maquinaria agrícola & $5(*)$ & 13 & $5(*)$ & $30(*)$ & 14 \\
\hline Textil, confecciones & 8 & 9 & $3(*)$ & $20(*)$ & 11 \\
\hline Servicios petroleros & $12(*)$ & 6 & 3 & 7 & 7 \\
\hline Embarcaciones & 8 & 5 & 2 & 5 & 5 \\
\hline Automotriz & 22 & 19 & $59(*)$ & $1(*)$ & 22 \\
\hline Siderurgia & 18 & $25(*)$ & 19 & $9(*)$ & 19 \\
\hline Industria de Morón & 26 & 23 & $10(*)$ & 27 & 22 \\
\hline Total & 100 & 100 & 100 & 100 & 100 \\
\hline
\end{tabular}

*Variables sobrerrepresentadas en el grupo (prueba Z con significación $p<0.05$ ).

Fuente: Elaboración propia con base en encuestas a ramas productivas argentinas.

En primer lugar, se observa que no existe ninguna rama particular identificada con la estrategia 1, lo que estaría implicando la existencia de un conjunto de empresas con dificultades para articular esfuerzos de innovación con independencia de la rama de pertenencia. ${ }^{14}$ En términos refinados, esto podría leerse como problemas asociados con el sistema de innovación como un todo, en donde pertenecer a un sector más dinámico en términos innovativos no compensa las dificultades previas.

En relación con la estrategia 2, el predominio de empresas de la rama siderúrgica se explica por sí misma. Por otra parte, el alto porcentaje de empre-

${ }^{14}$ Las empresas pertenecientes a la rama de servicios petroleros sobresale en este grupo; no obstante, es difícil establecer alguna conclusión debido tanto al poco número de observaciones de esa rama como a los problemas intrínsecos en la medición de la innovación en el sector servicios. 
sas que adoptan esta estrategia (casi 40\%) pone de manifiesto, en primer lugar, que durante los años recientes ha tenido lugar un proceso importante de incorporación de nueva maquinaria y, en segundo, que la incorporación de maquinaria es una de las estrategias predominantes de innovación de la industria local.

Las empresas automotrices destacan en el tercer grupo y las de maquinaria agrícola y confecciones en el cuarto. El componente de incorporación de software que caracteriza la cuarta estrategia podría explicar este comportamiento sectorial. Es razonable suponer que gran parte de las empresas pertenecientes a la rama automotriz ya realizaron en el pasado inversiones en software mientras que ramas como maquinaria agrícola y confecciones, que sólo recientemente alcanzaron un dinamismo considerable, estén en estos momentos ampliando su adopción de tecnologías de información y comunicación.

Cruzar las cuatro estrategias con los resultados de innovación (cuadro 5) permite corroborar la congruencia de las respuestas referidas a resultados y esfuerzos de innovación. A la vez, aporta nueva información que va en línea con la metodología utilizada.

Cuadro 5: Congruencia entre esfuerzos y resultados de innovación (porcentaje de la muestra)

\begin{tabular}{|c|c|c|c|c|c|c|}
\hline \multicolumn{2}{|c|}{$\begin{array}{l}\text { ¿Ha desarrollado } \\
\text { innovaciones... }\end{array}$} & $\begin{array}{l}\text { Esfuerzos } \\
\text { descoordinados o } \\
\text { falta de estrategia. }\end{array}$ & $\begin{array}{l}\text { Estrategia } \\
\text { basada en } \\
\text { adquisición de } \\
\text { bienes de capital. }\end{array}$ & $\begin{array}{l}\text { Estrategia basada } \\
\text { en nuevos } \\
\text { productos y } \\
\text { procesos. }\end{array}$ & $\begin{array}{l}\text { Estrategia basada en } \\
\text { nuevos productos y } \\
\text { procesos e inc. soft. }\end{array}$ & Total \\
\hline \multirow{3}{*}{$\begin{array}{r}\ldots \text { de } \\
\text { producto? }\end{array}$} & Sí & 50.0 & 69.4 & 83.9 & 80.4 & 70.8 \\
\hline & No & 50.0 & 30.6 & 16.1 & 19.6 & 29.2 \\
\hline & Total & 100.0 & 100.0 & 100.0 & 100.0 & 100.0 \\
\hline \multicolumn{7}{|c|}{ Pearson $\chi^{2}=24.4 p=0.0 \%$} \\
\hline \multirow{3}{*}{$\begin{array}{r}\ldots \mathrm{de} \\
\text { proceso? }\end{array}$} & Sí & 41.7 & 61.2 & 85.5 & 78.3 & 65.7 \\
\hline & No & 58.3 & 38.8 & 14.5 & 21.7 & 34.3 \\
\hline & Total & 100.0 & 100.0 & 100.0 & 100.0 & 100.0 \\
\hline \multicolumn{7}{|c|}{ Pearson $\chi^{2}=37.0 p=0.0 \%$} \\
\hline \multirow{3}{*}{$\begin{array}{r}\ldots \text { organiza- } \\
\text { cionales }\end{array}$} & Sí & 19.4 & 42.9 & 69.4 & 56.5 & 46.1 \\
\hline & No & 80.6 & 57.1 & 30.7 & 43.5 & 53.9 \\
\hline & Total & 100.0 & 100.0 & 100.0 & 100.0 & 100.0 \\
\hline \multicolumn{7}{|c|}{ Pearson $\chi^{2}=38.7 p=0.0 \%$} \\
\hline \multirow{3}{*}{$\begin{array}{r}\ldots \text { de } \\
\text { comercia- } \\
\text { lización? }\end{array}$} & Sí & 20.8 & 22.5 & 50.0 & 52.2 & 34.1 \\
\hline & No & 79.2 & 77.6 & 50.0 & 47.8 & 66.0 \\
\hline & Total & 100.0 & 100.0 & 100.0 & 100.0 & 100.0 \\
\hline \multicolumn{7}{|c|}{ Pearson $\chi^{2}=34.9 p=0.0 \%$} \\
\hline
\end{tabular}

Fuente: Elaboración propia con base en encuestas a ramas productivas argentinas. 
De este cruce surgen algunos elementos interesantes. En primer lugar, de acuerdo con la prueba chi cuadrada de Pearson, existen relaciones significativas entre esfuerzos y resultados. En segundo lugar, como era de esperar, las estrategias basadas en nuevos productos y procesos, con y sin incorporación de software, están asociadas con resultados de producto y proceso. En tercer lugar, en estos dos resultados de innovación hay menos diferencia cuando la estrategia es de compra de bienes de capital, y son casi independientes cuando no hay estrategia de innovación claramente identificada. En cuarto lugar, en el caso de resultados referidos a cambios organizacionales, las asociaciones son menos claras.

El 80\% de las empresas que no muestra una estrategia definida no obtuvo resultados en innovaciones organizacionales y, en cambio, en el otro extremo, las empresas que innovaron en proceso y producto con incorporación de software no muestran relación con el resultado en cambios organizacionales. En quinto término, esta situación se vuelve a poner de manifiesto en el caso de comercialización. Las firmas que no tienen estrategia definida -o quienes sostienen una estrategia basada en la compra de bienes de capital-, no innovaron en su comercialización, en tanto que las estrategias 3 y 4 se muestran independientes de los resultados en comercialización.

Las dos últimas observaciones ponen de manifiesto que no existe relación entre esfuerzos y resultados de innovación en los ámbitos "blandos" (organización y comercialización), o bien que la interpretación de los entrevistados sobre innovaciones en estos campos involucra mayores niveles de discrecionalidad, reafirmando nuestra elección metodológica.

Los resultados presentados hasta aquí muestran algunas pautas sobre las estrategias de innovación identificadas y el tipo de empresas que las seleccionan. Sin embargo, las pruebas de chi cuadrada pueden estar ocultando los efectos de variables excluidas. En la siguiente sección utilizaremos un modelo de regresión multinomial para identificar determinantes de las estrategias de una manera multivariada.

\section{Segunda etapa: Factores determinantes de las estrategias de innovación}

En el presente apartado se estima el impacto de los diferentes componentes de las capacidades de absorción y conectividad sobre la determinación de las estrategias de innovación que siguen las empresas. En este caso proponemos la utilización de un modelo logístico multinomial donde la variable dependiente (estrate- 
gia de innovación) sea una variable no ordinal. ${ }^{15}$ El cuadro 6 resume los resultados relativos a los indicadores de capacidad de absorción y conectividad por tipo de estrategia. En primer lugar, se observa cómo las dos primeras estrategias están fuertemente asociadas con bajas capacidades de absorción y con una proporción de conexiones inferior a la observada en las estrategias restantes. La estrategia 3, por su parte, se caracteriza por una alta capacidad de absorción y una conectividad sensiblemente superior con los tres tipos de agentes respecto a las otras tres estrategias. Finalmente, la estrategia basada en desarrollo de nuevos productos y procesos e incorporación de software se caracteriza por un nivel medio de capacidades de absorción y una conectividad superior a las dos primeras, pero inferior a la observada en la tercer estrategia, lo cual refuerza la idea de que los sectores pertenecientes a esa estrategia posiblemente hayan incorporado tecnologías de información y comunicación con anterioridad.

Cuadro 6. Estrategias de innovación y capacidades endógenas

\begin{tabular}{|c|c|c|c|c|c|c|}
\hline \multicolumn{2}{|c|}{ Estrategia de innovación } & $\begin{array}{l}\text { 1. Falta de } \\
\text { una } \\
\text { estrategia } \\
\text { clara de } \\
\text { innova- } \\
\text { ción. }\end{array}$ & $\begin{array}{l}\text { 2. Estrategia } \\
\text { basada en } \\
\text { la adqui- } \\
\text { sición de } \\
\text { bienes } \\
\text { de capital. }\end{array}$ & $\begin{array}{l}\text { 3. Estrategia } \\
\text { basada en la } \\
\text { investiga- } \\
\text { ción de } \\
\text { nuevos } \\
\text { productos y }\end{array}$ & $\begin{array}{l}\text { 4. Estrategia } \\
\text { basada en la } \\
\text { investigación } \\
\text { de nuevos } \\
\text { productos y } \\
\text { procesos e inc. }\end{array}$ & Total \\
\hline \multirow{3}{*}{$\begin{array}{l}\text { Capacidad } \\
\text { de } \\
\text { absorción. }\end{array}$} & Baja & $61(*)$ & $49(*)$ & 8 & 37 & 42 \\
\hline & Media & 29 & 29 & 33 & $42(*)$ & 33 \\
\hline & Alta & 10 & 22 & $58(*)$ & 19 & 25 \\
\hline \multirow{3}{*}{$\begin{array}{l}\text { Capacidad } \\
\text { de } \\
\text { conectivi- } \\
\text { dad. }\end{array}$} & Con otras empresas & 58 & 63 & $80\left(^{*}\right)$ & 68 & 65 \\
\hline & $\begin{array}{l}\text { Con instituciones } \\
\text { intermedias. }\end{array}$ & 34 & 41 & $70(*)$ & 53 & 47 \\
\hline & $\begin{array}{l}\text { Con universidades y } \\
\text { centros tec. }\end{array}$ & 26 & 35 & $63(*)$ & $60(*)$ & 44 \\
\hline
\end{tabular}

* Variables sub/sobrerrepresentadas en el grupo (prueba Z con significación $p<0.5$ ).

** Porcentaje de empresa con conexiones.

Fuente: Elaboración propia con base en encuestas a ramas productivas argentinas.

${ }^{15}$ Una variable se denomina nominal cuando las categorías que la componen no están ordenadas. El modelo de decisión multinomial representa las estrategias o esfuerzos de innovación de las empresas mediante una variable respuesta nominal. No obstante, puede darse el caso de que sea preferible considerar las estrategias de innovación de las empresas como una variable con características nominales aun cuando la misma se encuentre ordenada o parcialmente ordenada. $\mathrm{Si}$, por ejemplo, una estrategia de innovación particular invalida el concepto de orden jerárquico en la respuesta, entonces el modelo de respuesta ordinal para representar el proceso de elección de estrategia de innovación de las empresas es incorrecto. Para tal efecto, se considera un modelo de naturaleza no lineal para la variable respuesta nominal que rechaza por definición el supuesto de equidistancia entre las estrategias o esfuerzos de innovación. 
Los resultados de la estimación del modelo logístico pueden verse en el cuadro 7. La calidad de las vinculaciones con universidades y centros tecnológicos explica el pasaje hacia estrategias de innovación más complejas. De esta manera, este tipo de conexión resulta altamente significativo para explicar el paso desde la estrategia descoordinada a otras que implican mayores capacidades innovativas ( 3 y 4). En particular, es interesante notar cómo hay también una alta significación en esta variable para explicar el paso de la estrategia 4 a la 3 , lo cual una vez más refuerza la idea de mayor complejidad de la estrategia 3 . Ninguna otra dimensión de conectividad es significativa para explicar la decisión de la estrategia de innovación, lo cual podría estar revelando cierta actitud de aislamiento entre las empresas al interpretar que éstas pueden mostrar estrategias de diversa complejidad que se manifiestan en un marco de poca conectividad con clientes, proveedores, cámaras, consultores, etcétera.

Cuadro 7. Factores determinantes de las estrategias de innovación

\begin{tabular}{|c|c|c|c|c|c|c|}
\hline \multicolumn{2}{|c|}{$\begin{array}{l}\text { Paso de estrategias } \\
\text { Variables independientes }\end{array}$} & 1 a 2 & 1 a 3 & 1 a 4 & 2 a 3 & 3 a 4 \\
\hline \multirow{3}{*}{$\begin{array}{l}\text { Capacidades } \\
\text { de conectivi- } \\
\text { dad. }\end{array}$} & Empresas & + & + & + & + & - \\
\hline & Instituciones intermedias & - & - & - & - & + \\
\hline & $\begin{array}{l}\text { Universidades y centros } \\
\text { tecnológicos }\end{array}$ & + & $+(* *)$ & $+(* *)$ & $+(*)$ & $-(*)$ \\
\hline \multirow{3}{*}{$\begin{array}{l}\text { Capacidades } \\
\text { de } \\
\text { absorción. }\end{array}$} & Organización del trabajo & - & + & - & + & - \\
\hline & Gestión de la calidad & - & + & + & $+(* *)$ & - \\
\hline & $\begin{array}{l}\text { Capacitación } \\
\text { Equipo de ID }\end{array}$ & $\begin{array}{c}- \\
+(*)\end{array}$ & $\begin{array}{l}+(* * *) \\
+(* * * *)\end{array}$ & $\begin{array}{l}+(* *) \\
+(* *)\end{array}$ & $\begin{array}{c}+(* * * *) \\
+(* *)\end{array}$ & $\begin{array}{c}- \\
-(* *)\end{array}$ \\
\hline \multicolumn{2}{|c|}{ Rama productiva } & + & $+(* * *)$ & $-(* * *)$ & $+(*)$ & $-(* * *)$ \\
\hline \multicolumn{2}{|c|}{ Tamaño ( $L n$ empleo) } & + & + & + & + & + \\
\hline \multicolumn{2}{|c|}{ Tamaño ${ }^{2}\left(L n^{2}\right.$ empleo) } & - & - & - & - & + \\
\hline
\end{tabular}

$$
\begin{aligned}
* p & <0.1 . \\
* * & <0.05 . \\
* * * & <0.01 . \\
* * * * & <<0.001 .
\end{aligned}
$$

Número de obs. $=306 ; L R \chi^{2}(30)=156.14 ;$ prob. $>\chi^{2}=0.0000 ;$ pseudo $R^{2}=0.1942$.

Fuente: Elaboración propia con base en encuestas a ramas productivas argentinas. 
De igual forma, los componentes referidos a capacitación y existencia de equipos de ID, así como a gestión de la calidad (sólo en un caso) son estadísticamente relevantes para entender el paso desde estrategias desarticuladas hacia las de mayor complejidad relativa, en consonancia con lo planteado en la hipótesis 1. Nuevamente, el paso de la estrategia 4 a la 3 está asociado con una mayor importancia de los equipos de ID. Destaca la independencia de las estrategias de innovación respecto al indicador de organización del trabajo

Cabe mencionar que la pertenencia a diferentes ramas productivas también explica la elección de estrategias de innovación, mientras que el tamaño no aparece como una variable significativa.

\section{Tercera etapa: Impacto de las estrategias de innovación sobre el empleo}

En este apartado se analiza el efecto de las diferentes estrategias de innovación sobre cambios en el empleo y la calificación de los trabajadores, derivados de sus actividades de innovación. En esta última etapa se propone la utilización de un conjunto de modelos Probit que permitan mostrar cómo las diferentes estrategias repercuten sobre la existencia de cambios tanto en la cantidad como en la cantidad y calificación de los trabajadores. La utilización de modelos Probit aplicados a este problema nos permitirá estimar la probabilidad de incrementos en la cantidad de trabajadores así como en la calificación de los mismos ante la presencia de las diferentes estrategias de innovación posibles.

Para estimar cambios en el empleo, fueron utilizadas dos variables del cuestionario aplicado: $i$ ) si las actividades de innovación realizadas tuvieron impacto positivo sobre el monto del empleo (60\% de los casos que realizaron actividades de innovación), y ii) si estas actividades originaron un incremento en la calificación de sus trabajadores ( $64 \%$ de los casos). El uso de estas variables en vez de la variación total en el empleo permite desestimar el efecto del ciclo económico y dinámicas sectoriales estructurales sobre los cambios en el empleo (Evangelista y Savona, 2001).

Por otra parte, como puede verse en el cuadro 8 la variación total del empleo (durante el periodo 2001-2006) ${ }^{16}$ y la variable propuesta para estimar cambios en la cantidad del empleo asociado con innovación, muestran una elevada correlación. ${ }^{17}$

\footnotetext{
${ }^{16}$ Para los casos en que la información correspondiente a 2001 no existía se utilizó el dato correspondiente a 2003.

${ }^{17}$ Con $p>0.1 \%$, de acuerdo con la prueba de correlación de chi cuadrada de Pearson.
} 
Así, en los casos en los que el empresario declara que sus actividades de innovación llevaron a incrementar la planta de trabajadores, el empleo total efectivamente aumenta. No obstante, también se registraron casos en los que las empresas incrementaron su nivel de empleo a pesar de que sus actividades de innovación no implicaron nuevos puestos de trabajo (34\% de las empresas que subieron en su empleo).

Esto puede ser un indicio de que esos aumentos en la nómina se pueden deber a otras causas, como el ciclo económico o el desempeño sectorial. También están los casos opuestos, en los que a pesar de declarar incrementos en el empleo debidos a la innovación, no se registra un aumento en el total de la planta. En estos casos, el incremento debido a la innovación no llegaría a compensar la pérdida de empleo atribuible a otras causas.

Cuadro 8. Incremento en la cantidad y calificación de los trabajadores

\begin{tabular}{|c|c|c|c|c|c|c|}
\hline & \multicolumn{2}{|c|}{$\begin{array}{l}\text { a. ¿Requirió más trabajado- } \\
\text { res como resultado de sus } \\
\text { innovaciones? }\end{array}$} & \multicolumn{2}{|c|}{$\begin{array}{l}\text { b. ¿Requirió trabajadores con } \\
\text { mayores calificaciones } \\
\text { como resultado de sus } \\
\text { innovaciones? }\end{array}$} & \multirow{3}{*}{$\begin{array}{c}\text { Total } \\
61\end{array}$} \\
\hline & & Sí & No & Sí & No & \\
\hline \multirow{3}{*}{$\begin{array}{l}\text { ¿Aumentó el número de } \\
\text { empleados entre } 2001 \text { y } \\
2006 ?\end{array}$} & Sí & 19 & 42 & 26 & 35 & \\
\hline & No & 163 & 82 & 168 & 82 & 245 \\
\hline & Total & 182 & 124 & 194 & 124 & 306 \\
\hline \multirow{3}{*}{\multicolumn{2}{|c|}{$\begin{array}{l}\text { Impacto positivo en la cantidad } \\
\text { del empleo debido a la } \\
\text { innovación. }\end{array}$}} & \multicolumn{2}{|c|}{$\begin{array}{c}\text { Pearson } \chi^{2}=25.4 \\
p=0.0 \%\end{array}$} & \multicolumn{2}{|c|}{$\begin{array}{c}\text { Pearson } \chi^{2}=14.2 \\
\quad p=0.0 \%\end{array}$} & \\
\hline & & \multicolumn{2}{|c|}{$\begin{array}{l}\text { a. ¿Requirió más trabajado- } \\
\text { res como resultado de sus } \\
\text { innovaciones? }\end{array}$} & \multicolumn{2}{|c|}{$\begin{array}{l}\text { b. ¿Requirió trabajadores con } \\
\text { mayores calificaciones } \\
\text { como resultado de sus } \\
\text { innovaciones? }\end{array}$} & \\
\hline & & Sí & No & Sí & No & Total \\
\hline \multirow{3}{*}{$\begin{array}{l}\text { ¿Aumentó el número de } \\
\text { empleados entre } 2001 \text { y } \\
\text { 2006? }\end{array}$} & Sí & $10 \%$ & $34 \%$ & $13 \%$ & $31 \%$ & $20 \%$ \\
\hline & No & $90 \%$ & $66 \%$ & $87 \%$ & $69 \%$ & $80 \%$ \\
\hline & Total & $100 \%$ & $100 \%$ & $100 \%$ & $100 \%$ & $100 \%$ \\
\hline \multicolumn{2}{|c|}{$\begin{array}{l}\text { Impacto positivo en la cantidad } \\
\text { del empleo debido a la } \\
\text { innovación. }\end{array}$} & \multicolumn{2}{|c|}{$\begin{array}{l}\text { Pearson } \chi^{2}=25.4 \\
p=0.0 \%\end{array}$} & \multicolumn{2}{|c|}{$\begin{array}{l}\text { Pearson } \chi^{2}=14.2 \\
p=0.0 \%\end{array}$} & \\
\hline
\end{tabular}

Fuente: Elaboración propia con base en encuestas a ramas productivas argentinas. 
El cuadro 8 muestra una relación interesante entre incremento en la cantidad y calificación de los trabajadores y la variación positiva en el total del empleo de la empresa. ${ }^{18}$ Los resultados de esta asociación están en consonancia con la hipótesis 3, en la que afirmamos que cambios en la calificación de los trabajadores se explican por cambios en la cantidad. De corroborarse esta hipótesis podemos afirmar que los nuevos puestos de trabajo debidos a la innovación requieren mayores calificaciones o, lo que es similar, que las empresas prefieren contratar a nuevos trabajadores más calificados que realizar una capacitación interna cuando las innovaciones demandan cambios en la calificación de los trabajadores.

Por otra parte, como puede verse en las estadísticas descriptivas (cuadro 9), las empresas que siguieron las estrategias 3 y 4 están sobrerrepresentadas entre las que tuvieron impacto positivo de la innovación sobre el empleo, mientras que en lo que se refiere a cambios en la calificación de los trabajadores sólo las empresas que siguieron la estrategia 4 muestran esta sobrerrepresentación. ${ }^{19}$

Cuadro 9. Estrategia de innovación e impacto de la innovación

\begin{tabular}{|c|c|c|c|c|c|c|}
\hline \multicolumn{2}{|c|}{$\begin{array}{l}\text { Estrategia de innovación } \\
\text { Variable independiente }\end{array}$} & $\begin{array}{l}\text { 1. Falta de } \\
\text { una } \\
\text { estrategia } \\
\text { clara de } \\
\text { innova- } \\
\text { ción. }\end{array}$ & $\begin{array}{l}\text { 2. Estrategia } \\
\text { basada } \\
\text { en la } \\
\text { adquisición } \\
\text { de bienes } \\
\text { de capital. }\end{array}$ & $\begin{array}{l}\text { 3. Estrategia } \\
\text { basada } \\
\text { en la } \\
\text { investiga- } \\
\text { ción de } \\
\text { nuevos } \\
\text { productos y } \\
\text { procesos. }\end{array}$ & $\begin{array}{l}\text { 4. Estrategia } \\
\text { basada en la } \\
\text { investigación } \\
\text { de nuevos } \\
\text { productos y } \\
\text { procesos e } \\
\text { inc. soft. }\end{array}$ & Total \\
\hline \multirow{2}{*}{$\begin{array}{l}\text { Impacto } \\
\text { positivo de la } \\
\text { innovación } \\
\text { en ... }\end{array}$} & $\begin{array}{c}\text {... la cantidad } \\
\text { de trabajadores }\end{array}$ & 38 & 60 & 61 & $71\left(^{*}\right)$ & 60 \\
\hline & $\begin{array}{l}\ldots . . \text { las califica- } \\
\text { ciones de los } \\
\text { trabajadores }\end{array}$ & 36 & 55 & $81\left(^{*}\right)$ & $83\left(^{*}\right)$ & 64 \\
\hline
\end{tabular}

*Variables sobre/subrepresentadas en el grupo (la prueba Z con significación $p<0.5$ ).

Fuente: Elaboración propia con base en encuestas a ramas productivas argentinas.

Los resultados de la estimación de los modelos Probit que muestran la incidencia de las estrategias sobre cambios en el empleo están plasmados en el cuadro 10. Como puede verse, las distintas estrategias de innovación seguidas por las empresas tienen diferente impacto no sólo en la creación de puestos de

\footnotetext{
${ }^{18}$ Con $p>0.1 \%$, de acuerdo con la prueba de correlación de chi cuadrada de Pearson.

${ }^{19}$ Cabe mencionar que en todos los casos las diferencias son estadísticamente significativas de acuerdo con la prueba.
} 
trabajo asociados con la innovación, sino también en el nivel de calificación de los trabajadores.

En este caso, las estrategias 2, 3 y 4 muestran un impacto positivo sobre la cantidad de empleo, en comparación con la estrategia 1. Mientras sólo las estrategias 3 y 4 demandan trabajadores de mayor calificación, no hay diferencias entre las estrategias 1 y 2 en lo que respecta a la calificación de los trabajadores. Esto significa que, de acuerdo con nuestra información empírica, la estrategia basada en la compra de bienes de capital no demanda cambios en la calificación de los trabajadores.

\section{Cuadro 10. Estrategias de innovación y cambios en el empleo}

\begin{tabular}{|c|c|c|c|}
\hline \multicolumn{2}{|c|}{$\begin{array}{l}\text { Variables dependientes } \\
\text { Variables independientes }\end{array}$} & $\begin{array}{l}\text { Impacto positivo en la } \\
\text { cantidad del empleo. }\end{array}$ & $\begin{array}{l}\text { Impacto positivo en la } \\
\text { calificación del empleo. }^{\text {b }}\end{array}$ \\
\hline \multirow{3}{*}{$\begin{array}{l}\text { Estrategia de } \\
\text { innovación }\end{array}$} & $\begin{array}{l}\text { 2. Estrategia basada en la } \\
\text { adquisición de bienes } \\
\text { de capital. }\end{array}$ & $+(* * *)$ & + \\
\hline & $\begin{array}{l}\text { 3. Estrategia basada en la } \\
\text { investigación de nuevos } \\
\text { productos y procesos. }\end{array}$ & $+(* *)$ & $+(*)$ \\
\hline & $\begin{array}{l}\text { 4. Estrategia basada en la } \\
\text { investigación de nuevos } \\
\text { productos y procesos e } \\
\text { inc. soft. }\end{array}$ & $+(* * *)$ & $+(* * *)$ \\
\hline \multicolumn{2}{|c|}{$\begin{array}{l}\text { Impacto positivo en la cantidad del } \\
\text { empleo debido a la innovación. }\end{array}$} & & $+(* * * *)$ \\
\hline \multicolumn{2}{|c|}{ Capacidades de absorción. } & + & $+(* *)$ \\
\hline \multicolumn{2}{|c|}{ Rama productiva } & $-(*)$ & $-(*)$ \\
\hline \multicolumn{2}{|c|}{ Tamaño (Ln empleo) } & + & + \\
\hline \multicolumn{2}{|c|}{ Tamaño ${ }^{2}$ ( $L n^{2}$ empleo) } & + & - \\
\hline
\end{tabular}

\footnotetext{
$* p<0.1$.

$* * p<0.05$.

$* * * p<0.01$.

$* * * * p<0.001$.

Numerario: Estrategia 1.

a Núm. de obs. $=306 ; L R \chi^{2}=28.68 ; p$ value $=0.0002 ;$ pseudo $R^{2}=0.0692$

${ }^{\text {b }}$ Núm. de obs. $=306 ; L R \chi^{2}=95.26 ; p$ value $=0.0000 ;$ pseudo $R^{2}=0.2335$

Fuente: Elaboración propia con base en encuestas a ramas productivas argentinas.
} 
Por otra parte, haber tenido impacto positivo en la cantidad de trabajadores está asociado positivamente con impactos positivos en la calificación de los trabajadores, lo que demuestra que prima la incorporación de trabajadores de mayor calificación sobre la capacitación de trabajadores. Por otra parte, existe un impacto positivo de las capacidades de absorción sobre el nivel de calificación de los trabajadores, aunque no es así para el caso de variaciones en la cantidad. Esto implica que mientras en el primer caso existe un impacto directo e indirecto (a partir de las estrategias de innovación) de la capacidad de absorción, para el segundo es sólo indirecto. Por tanto, las políticas que tiendan a incrementar el nivel de capacidades de absorción de las empresas sólo podrían resultar en cambios en la cantidad de empleo por el efecto que estas capacidades tienen sobre la elección de estrategias de las empresas.

Dos últimas cuestiones merecen ser mencionadas. En primer lugar, que las diferentes ramas productivas tienen un efecto diferente sobre los cambios en cantidad y calificación de los trabajadores. Esto también aporta elementos clave para el diseño de política porque no en todos lo casos la respuesta sobre el empleo es la misma. En segundo lugar, el tamaño de la organización no parece desempeñar un papel clave para explicar los cambios en el empleo.

\section{CONCLUSiOnes}

Este artículo retoma la larga discusión al interior de la teoría económica acerca de la relación entre innovación y dinámica del empleo. En la línea de una perspectiva evolucionista y neoschumpeteriana, ampliada por la teoría de los sistemas complejos, se plantea que los procesos de aprendizaje de las empresas están sujetos a dinámicas no lineales y que los resultados de las actividades de innovación deben ser vistos como una propiedad emergente en la que son clave los niveles y dinámicas alcanzados por las capacidades de absorción y conectividad de las empresas acumuladas a lo largo de sus senderos de aprendizaje.

Es decir, se considera que las actividades de innovación de las empresas no se generan en forma desarticulada sino en el marco de estrategias que combinan esfuerzos diversos y que están limitadas y condicionadas por las capacidades mencionadas y por las oportunidades existentes en la rama productiva en la que operan. Las capacidades no sólo tienen un efecto indirecto sobre la generación de empleo a partir de su impacto sobre las estrategias de innovación, sino también un efecto directo, dado que las empresas de mayores capacidades pueden mejorar su posición competitiva y expandir su tamaño. En esa dirección se 
sostiene que los cambios en la calidad y cantidad de empleo dependen de la estrategia innovativa elegida por las empresas más que de los efectos independientes y de distinto signo que tienen las innovaciones de producto y de proceso consideradas en forma aislada.

Se parte así de un marco teórico que, para estudiar el efecto que tienen las estrategias de innovación y las capacidades de las empresas que determinan su dinámica de la cantidad y calidad del empleo asociada con la innovación, combina el enfoque evolucionista con el de sistemas complejos, tomando de éste las ideas de emergencia y realimentación entre variables.

El trabajo identifica diferentes estrategias de innovación y muestra, a partir de un análisis de regresión multinomial, que estas estrategias están fuertemente influidas por las capacidades que alcanzan. A su vez, utilizando modelos Probit se muestra que tanto las estrategias de innovación identificadas como la capacidad de absorción influyen en las variaciones en la cantidad y la calidad del empleo asociadas con las actividades de innovación.

Este conjunto de cuestiones se analiza en una muestra de 403 empresas pertenecientes a siete ramas productivas argentinas. Un primer resultado del trabajo es haber identificado cuatro estrategias de innovación cuyo grado de complejidad depende de las capacidades de absorción y conectividad de las empresas. La mayor parte de las empresas o bien carece de una estrategia (20\%) o tiene una estrategia centrada sólo en la compra de bienes de capital (40\%). El resto tiene estrategias más complejas, identificadas por el desarrollo de productos y procesos con sesgo hacia actividades de investigación y desarrollo en un caso (15\%) y desarrollo de software en el otro (25\%). Las dos primeras estrategias identificadas están fuertemente asociadas con bajas capacidades de absorción y con conexiones de menor calidad que en los dos grupos restantes. La tercera estrategia se caracteriza por una alta capacidad de absorción y una conectividad sensiblemente superior respecto a las otras tres estrategias. Finalmente, la cuarta estrategia se caracteriza por un nivel medio de capacidades de absorción y una conectividad superior a las dos primeras pero inferior a la observada en la tercera estrategia.

Pensamos que si bien el análisis se realiza en el nivel de empresas individuales, el control que se efectúa por el efecto de la pertenencia a una rama es clave para poder limitar el efecto de los mecanismos de compensación discutidos por la bibliografía, dado que no sólo están incluidas las empresas del sector al que cada agente pertenece sino también las empresas vinculadas por relaciones de compra-venta con los núcleos de las ramas estudiadas. El artículo corrobora el marco teórico presentado y muestra que las capacidades de absorción de las 
empresas influyen tanto en forma indirecta como directa sobre el empleo generado. La influencia indirecta se centra en el efecto que las capacidades tienen sobre las estrategias de innovación. A su vez, la influencia directa se explica por el efecto de las capacidades de absorción en la posición competitiva de las empresas y, por tanto, en la posibilidad de expandir su tamaño y generar en consecuencia más empleo. El trabajo muestra a su vez que los aspectos sectoriales también son significativos, poniendo de manifiesto una dimensión que va más allá de las respuestas individuales de las empresas.

\section{Cuadro 11. Resumen de los resultados obtenidos}

\begin{tabular}{|c|c|c|}
\hline \multicolumn{2}{|c|}{ Hipótesis de trabajo } & Corroborada \\
\hline \multicolumn{2}{|c|}{$\begin{array}{l}\text { H1a. Existe una relación de causalidad entre el nivel alcan- } \\
\text { zado por las capacidades de las empresas y la estrategia de } \\
\text { innovación. }\end{array}$} & $\begin{array}{l}\text { * Sólo algunos componentes de la ca- } \\
\text { pacidad de absorción explican las es- } \\
\text { trategias. Sólo las vinculaciones con } \\
\text { universidades y centros tecnológicos } \\
\text { explican las estrategias. }\end{array}$ \\
\hline \multicolumn{2}{|c|}{$\begin{array}{l}\text { H1b. Mayores capacidades adquiridas se corresponden con } \\
\text { estrategias más sofisticadas de innovación. }\end{array}$} & $\begin{array}{l}\checkmark \text { Las estrategias } 3 \text { y } 4 \text { requieren mayo- } \\
\text { res capacidades de absorción y conec- } \\
\text { tividad. }\end{array}$ \\
\hline \multirow[t]{2}{*}{$\begin{array}{l}\text { H2. Los cambios en la canti- } \\
\text { dad de trabajadores se en- } \\
\text { cuentran causalmente aso- } \\
\text { ciados con... }\end{array}$} & $\begin{array}{l}\text {... el tipo de estrategia de } \\
\text { innovación. }\end{array}$ & $\begin{array}{l}\checkmark \text { Las estrategias } 2,3 \text { y } 4 \text { implicaron in- } \\
\text { crementos en la cantidad de trabaja- } \\
\text { dores, en relación con la estrategia } 1 .\end{array}$ \\
\hline & $\begin{array}{l}\ldots \text { las capacidades de ab- } \\
\text { sorción acumuladas. }\end{array}$ & $\begin{array}{l}\text { * Las capacidades de absorción no } \\
\text { muestran un efecto directo sobre } \\
\text { cambios en el empleo asociados con } \\
\text { la innovación, sólo indirecto a partir } \\
\text { de las estrategias de innovación. }\end{array}$ \\
\hline \multirow[t]{3}{*}{$\begin{array}{l}\text { H3. Los cambios en la califi- } \\
\text { cación de los trabajadores } \\
\text { se encuentra causalmente } \\
\text { asociada con... }\end{array}$} & $\begin{array}{l}\text {... el tipo de estrategia de } \\
\text { innovación. }\end{array}$ & $\begin{array}{l}\text { × Sólo las estrategias } 3 \text { y } 4 \text { implicaron } \\
\text { incrementos en la calificación de los } \\
\text { trabajadores en relación con la estra- } \\
\text { tegia } 1 .\end{array}$ \\
\hline & $\begin{array}{l}\text {.. las capacidades de absor- } \\
\text { ción acumuladas. }\end{array}$ & $\begin{array}{l}\text { El grado de desarrollo de capacidades } \\
\text { de absorción repercute directamente } \\
\text { sobre la dinámica en la calificación de } \\
\text { los trabajadores. }\end{array}$ \\
\hline & $\begin{array}{l}\text {.. los incrementos en la } \\
\text { cantidad de trabajadores } \\
\text { derivados de la innovación. }\end{array}$ & $\begin{array}{l}\checkmark \text { El incremento en la cantidad de traba- } \\
\text { jadores conduce a incrementos en la } \\
\text { calificación de los trabajadores. }\end{array}$ \\
\hline \multicolumn{2}{|c|}{$\begin{array}{l}\text { H4. Existen efectos sectoriales en los cambios en el empleo } \\
\text { (cantidad y calificación) debido a la innovación. }\end{array}$} & $\begin{array}{l}\checkmark \quad \text { Las distintas ramas muestran dinámi- } \\
\text { cas específicas en el empleo. }\end{array}$ \\
\hline
\end{tabular}

Fuente: Elaboración propia con base en encuestas a ramas productivas argentinas. 
El trabajo corrobora mayormente las cuatro hipótesis que se desprenden del marco teórico presentado. En primer lugar, el grado de complejidad de las estrategias de innovación seguidas por las empresas está fuertemente asociado con sus capacidades de absorción y de conectividad. Así, el análisis multinomial revela que las empresas en las que prevalece la ausencia de estrategia, en las que no destaca ninguna rama, son las que tienen menores capacidades de absorción. En el otro extremo, las empresas con las estrategias de mayor complejidad relativa son las que lograron mayor articulación entre esfuerzos incorporados y desincorporados de innovación y las que tienen mayores capacidades de absorción. Mientras las empresas automotrices destacan en el tercer grupo, las de maquinaria agrícola y confecciones sobresalen en el cuarto. Asimismo, la presencia de capacitación, la existencia de equipos de ID y la gestión de la calidad permiten explicar el paso desde estrategias desarticuladas hacia las de mayor complejidad relativa.

En segundo lugar, la variación del empleo de las empresas durante el periodo analizado depende positivamente de las capacidades de absorción acumuladas y del tipo de estrategia identificada. En este caso, tener algún tipo de estrategia innovativa, en relación con las empresas que carecen de ellas, tiene un impacto positivo sobre la dinámica del empleo. Por su parte, sólo las estrategias de innovación de mayor complejidad relativa demandan trabajadores de mayor calificación. Los resultados de esta asociación están en consonancia con la hipótesis que sostiene que los cambios en la calificación de los trabajadores se explican por cambios en la cantidad de trabajadores demandados derivados del desarrollo de actividades de innovación. En tal sentido, la asociación entre la variación de la cantidad de ocupados y la calidad de los puestos revela que las empresas prefieren contratar a nuevos trabajadores más calificados que generar procesos de capacitación internos cuando las innovaciones desarrolladas demandan cambios en la calificación de los trabajadores. El trabajo también muestra que el tamaño de la organización no parece jugar un papel clave para explicar los cambios en el empleo derivados del desarrollo de actividades innovativas.

Finalmente, del trabajo se desprenden algunas recomendaciones de política industrial y tecnológica activas que rompen con la idea de que cada empresa maximiza su estrategia dada una restricción tecnológica o de mercado. Puesto que las estrategias de innovación más débiles están asociadas con sectores poco dinámicos y con empresas con bajas capacidades, las recomendaciones apuntan a fortalecer las capacidades endógenas de las empresas y el enramado productivo en el que operan, y a lograr una mayor complejidad del perfil productivo con 
mayor participación en sectores dinámicos. Es decir, una dinámica más positiva del empleo en términos de cantidad y calidad de los puestos generados requiere fortalecer las capacidades de absorción y conectividad de las empresas, las que influyen sobre el empleo a partir de una mejora en las estrategias de innovación y en una mejor posición competitiva en los mercados.

\section{Anexo Metodológico}

\section{A1. Construcción de indicadores de absorción y conectividad}

Los niveles de las capacidades de absorción y conectividad fueron definidos a partir de un conjunto de dimensiones que dan cuenta de las competencias de las empresas y de la calidad de las vinculaciones que éstas establecen con diferentes organizaciones pertenecientes a las ramas. El índice de capacidades de absorción es el promedio simple de cuatro indicadores:

1. Gestión de la calidad. El indicador de gestión de calidad, en línea con lo propuesto en la sección teórica del trabajo, combina un conjunto de dimensiones asociadas con la calidad: $i$ ) el control de calidad de procesos y productos; $i i$ ) las prácticas de mejora continua, y iii) la cultura empresarial frente a la calidad.

i) Control de procesos. Se definió un indicador a partir del grado de aplicación de los siguientes ítems referidos al control de productos y/o procesos: definición de requisito del producto, control de características del producto, documentación de actividades de proceso, controles de proceso, registro de datos en formularios, archivo de datos registrados, especificaciones de materias primas e insumos críticos, rastreabilidad y calibración de equipos.

ii) Cultura hacia la calidad. Se definió considerando la existencia y desarrollo de: comunicaciones periódicas con clientes, política de calidad, definición de indicadores clave, equipos de solución de problemas, auditoría interna, sistema de orden y limpieza y sistema de reconocimiento de ideas.

iii) Utilización de métodos sistemáticos para la mejora y la innovación. Se estimó a partir del uso de herramientas y métodos sistemáticos de análisis orientados a la mejora y la innovación (histogramas, diagrama de causa y efecto, AMFE, diagrama de Pareto, y gráficos de control esta- 
dístico de procesos, entre otros) y el nivel de participación de los empleados en la utilización de las mismas.

2. Capacitación de la mano de obra. El entrenamiento como medio de adquisición de conocimiento hace referencia a las acciones implantadas para inducir el aprendizaje de los trabajadores y, desde allí, incrementar las capacidades endógenas de la empresa. La capacitación es clave en los procesos de aprendizaje al permitir la integración entre conocimiento tácito y codificado. El indicador puede tomar tres valores: 1) si la empresa no capacita a sus empleados; 2) si lo hace con una estructura tercerizada, y 3) si lo hace y tiene estructura interna de capacitación.

3. Organización del trabajo. Este indicador considera un conjunto de aspectos técnicos y sociales que intervienen en el proceso productivo. El indicador agregado surge de la combinación de la flexibilidad de los puestos (rotación) y la conformación de equipos de trabajo.

4. Grupos de ID. Finalmente, se construyó un cuarto indicador a partir de la existencia y grado de formalidad de los departamentos de ID, como un elemento clave para definir el grado de absorción de las empresas. El indicador puede tomar tres valores: 1) si la empresa no tiene departamento de ID; 2) si cuenta con uno pero tiene una estructura informal, y 3) si el departamento es formal (en el sentido de tener personal asignado, tareas definidas, espacios, etcétera).

El rango de cada indicador varía de 1 a 3 . El indicador agregado de capacidades de absorción toma tres valores: bajo (de 1 a 1.85), medio (1.85 a 2.4) y alto (2.4 a 3$)$.

Para evaluar la capacidad de conectividad de las empresas se elaboraron tres índices de calidad de la vinculación de acuerdo con las vinculaciones establecidas con tres tipos distintos de socios: $i$ ) otras empresas (clientes, proveedores o competidores); $i$ ) cámaras de comercio y consultores, y iii) universidades o centros tecnológicos y científicos.

Al evaluar la calidad de la vinculación con un tipo de socio fueron tomados en cuenta los objetivos involucrados en el vínculo, considerando vinculaciones de mayor calidad aquellas que más objetivos involucran. Los objetivos considerados en el formulario fueron: $i$ ) objetivos comerciales; $i i)$ certificación de calidad; iii) capacitación de RRHH, iv) actividades de diseño y desarrollo; 
v) financiamiento para la innovación; vi) utilización conjunta de infraestructura; vii) reducir costos y riesgos de innovación; viii) cambios organizacionales, y ix) mejoras ambientales.

Los tres indicadores varían entre 1 y 4 , tomando el nivel 1 en caso de ausencia de vinculaciones, el 2 para las vinculaciones de baja calidad (tan sólo un objetivo), el 3 para vinculaciones de calidad media (dos o tres objetivos) y el 4 para vinculaciones de alta calidad (más de tres objetivos).

\section{A2. Cuestionario utilizado}

El cuestionario utilizado constó de cinco secciones. La primera indagaba datos generales de la empresa entrevistada (como tamaño, origen del capital, rama de actividad, etcétera); la segunda, las vinculaciones (extracomerciales) que establecía la empresa con otras organizaciones. La tercera sección estaba dedicada a la recolección de información sobre la conducta innovativa de las empresas, mientras que las secciones cuarta y quinta indagaban acerca de las formas de organización del trabajo y de las actividades de capacitación emprendidas por la empresa.

A continuación se reproducen las preguntas del cuestionario aplicado que fueron utilizadas en la construcción de estos indicadores y otras variables empleadas en el artículo.

\begin{tabular}{|l|l|l|l|}
\hline $\begin{array}{l}\text { Objetivo de la } \\
\text { vinculación con... }\end{array}$ & Otras empresas & $\begin{array}{l}\text { Cámaras y } \\
\text { consultores. }\end{array}$ & $\begin{array}{l}\text { Universidades y } \\
\text { centro de cyт. }\end{array}$ \\
\hline $\begin{array}{l}\text { Objetivos } \\
\text { comerciales. }\end{array}$ & & & \\
\hline $\begin{array}{l}\text { Certificación de } \\
\text { calidad. }\end{array}$ & & & \\
\hline Capacitación de RRH. & & & \\
\hline $\begin{array}{l}\text { Actividades de diseño } \\
\text { y desarrollo. }\end{array}$ & & & \\
\hline $\begin{array}{l}\text { Financiamiento para } \\
\text { la innovación. }\end{array}$ & & & \\
\hline $\begin{array}{l}\text { Utilizar/compartir } \\
\text { infraestructura. }\end{array}$ & & & \\
\hline
\end{tabular}


148 ECONOMÍA: TEORÍA Y PRÁCTICA • Nueva Época, número 32, enero-junio 2010

\begin{tabular}{|l|l|l|l|}
\hline $\begin{array}{l}\text { Objetivo de la } \\
\text { vinculación con... }\end{array}$ & Otras empresas. & $\begin{array}{l}\text { Cámaras y } \\
\text { consultores. }\end{array}$ & $\begin{array}{l}\text { Universidades y } \\
\text { centro de cyт. }\end{array}$ \\
\hline $\begin{array}{l}\text { Reducir costos y } \\
\text { riesgos de innovación. }\end{array}$ & & & \\
\hline $\begin{array}{l}\text { Cambios } \\
\text { organizacionales. }\end{array}$ & & & \\
\hline $\begin{array}{l}\text { Mejoras } \\
\text { ambientales. }\end{array}$ & & & \\
\hline
\end{tabular}

¿Ha destinado esfuerzos y/o recursos a actividades innovativas y de inversión en... (sí/no)?

\begin{tabular}{|l|l|l|}
\hline $\begin{array}{l}\text { Compra de bienes de capital } \\
\text { relacionados con prod. y/o proc. } \\
\text { nuevos o mejorados. }\end{array}$ & $\begin{array}{l}\text { Capacitación orientada } \\
\text { a la innovación. }\end{array}$ & $\begin{array}{l}\text { Investigación o explora- } \\
\text { ción de oportunidades } \\
\text { científicas. }\end{array}$ \\
\hline Desarrollo de software. & Consultoría. & $\begin{array}{l}\text { Programas de mejora } \\
\text { continua. }\end{array}$ \\
\hline $\begin{array}{l}\text { Diseño y desarrollo de canales } \\
\text { de comercialización. }\end{array}$ & $\begin{array}{l}\text { Diseño y desarrollo } \\
\text { de producto. }\end{array}$ & $\begin{array}{l}\text { Adquisición } \\
\text { de licencias. }\end{array}$ \\
\hline Cambio organizacional. & Desarrollo de procesos. & $\begin{array}{l}\text { Incorporación } \\
\text { de software. }\end{array}$ \\
\hline
\end{tabular}

Control de proceso y/o producto o servicio (sí/no)

Especificaciones de materias primas e insumos críticos.

Identificación de características críticas del proceso, producto y/o servicio.

Documentación de características críticas del proceso, producto y/o servicio.

Control de características críticas del proceso, producto y servicio.

Trazabilidad. 


\begin{tabular}{|c|c|}
\hline $\begin{array}{l}\text { Política de calidad definida y comunicada al } \\
\text { personal. }\end{array}$ & $\begin{array}{l}\text { Política de calidad definida y comunicada al } \\
\text { personal. }\end{array}$ \\
\hline $\begin{array}{l}\text { Sistema de reconocimiento de ideas o aporta- } \\
\text { ciones destacados. }\end{array}$ & Auditorías internas. \\
\hline $\begin{array}{l}\text { Uso de las comunicaciones con clientes para } \\
\text { modificar el producto o servicio. }\end{array}$ & $\begin{array}{l}\text { Comunicaciones periódicas con clientes para } \\
\text { detectar cambios en los requerimientos. }\end{array}$ \\
\hline $\begin{array}{l}\text { Sistema para mantener el orden y la } \\
\text { limpieza. }\end{array}$ & Comunicaciones periódicas con proveedores \\
\hline $\begin{array}{l}\text { Uso de las comunicaciones con proveedores } \\
\text { para modificar el producto o servicio. }\end{array}$ & $\begin{array}{l}\text { Equipos para solucionar problemas o lograr } \\
\text { mejoras en el proceso. }\end{array}$ \\
\hline
\end{tabular}

Herramientas y métodos sistemáticos para la mejora y la innovación.

\begin{tabular}{|l|l|}
\hline Gráficos de control estadístico de procesos. & Diagrama de Pareto. \\
\hline Histogramas. & AMFE/FMEA. \\
\hline Diagrama de causa-efecto. & Otros. \\
\hline
\end{tabular}

Modalidades de rotación del personal (sí/no/planeada/espontánea)

Rotación entre puestos de complejidad similar.

Rotación entre funciones de diferente complejidad.

Rotación entre diferentes áreas. 
150 ECONOMÍA: TEORÍA Y PRÁCTICA • Nueva Época, número 32, enero-junio 2010

Grado de flexibilidad predominante en la asignación de tareas en la gestión productiva (sí/no)

Trabajo individual y se le asignan a las mismas personas las mismas tareas. Puestos fijos.

Trabajo individual y se rota regularmente a las personas en los distintos puestos.

Trabajo en células/equipos y puestos fijos dentro de cada equipo.

Trabajo en células/equipos y dentro de cada célula la gente tiene rotación.

Otro (especificar).

¿Ha experimentado cambios en el empleo como consecuencia de los resultados de innovación? (sí/no)

Requirió más trabajadores.

Requirió menos trabajadores.

Requirió trabajadores con mayores calificaciones.

Requirió trabajadores con menores calificaciones. 


\section{ANEXO ESTAdíSTICO}

Cuadro A1. Distribución de empresas por cantidad de empleados

\begin{tabular}{|l|c|c|c|c|}
\hline \multirow{2}{*}{ Empleados } & \multicolumn{2}{|c|}{ Empresas } & \multicolumn{2}{c|}{ Empleados } \\
\cline { 2 - 5 } & Total & Porcentaje & Total & Porcentaje \\
\hline 20 o menos & 141 & 36 & 1530 & 4 \\
\hline 21 a 50 & 85 & 22 & 2803 & 8 \\
\hline 51 a 100 & 82 & 21 & 6444 & 18 \\
\hline 101 a 500 & 72 & 18 & 16254 & 46 \\
\hline Más de 500 & 12 & 3 & 7995 & 23 \\
\hline Total & 392 & 100 & 35026 & 100 \\
\hline
\end{tabular}

Fuente: Elaboración propia con base en encuestas a ramas productivas argentinas.

\section{Cuadro A2. Distribución de las empresas según destino de sus ventas}

\begin{tabular}{|r|c|c|r|r|}
\hline \multicolumn{4}{|c|}{ a. Coeficiente de exportación } \\
\hline \multirow{2}{*}{$\begin{array}{c}\text { ¿Sus ventas tienen } \\
\text { como destino el... }\end{array}$} & \multicolumn{1}{|c|}{ Sí mercado externo? } \\
\cline { 2 - 5 } & Sí & $28 \%$ & $0 \%$ & $16 \%$ \\
\hline \multirow{2}{*}{$\begin{array}{r}\text { mercado } \\
\text { interno? }\end{array}$} & No & $100 \%$ & $100 \%$ & $100 \%$ \\
\cline { 2 - 5 } & Total & $32 \%$ & $0 \%$ & $19 \%$ \\
\hline
\end{tabular}

\begin{tabular}{|r|c|c|c|c|}
\hline \multicolumn{4}{|c|}{ b. Cantidad de empresas } \\
\hline \multirow{2}{*}{$\begin{array}{c}\text { ¿Sus ventas tienen } \\
\text { como destino el... }\end{array}$} & \multicolumn{1}{|c|}{ Sí } & No mercado externo? & Total \\
\cline { 2 - 5 } & Sí & $55 \%$ & $43 \%$ & $97 \%$ \\
\cline { 2 - 5 } & No & $3 \%$ & $0 \%$ & $3 \%$ \\
\cline { 2 - 5 } & Total & $57 \%$ & $43 \%$ & $100 \%$ \\
\hline
\end{tabular}

Fuente: Elaboración propia con base en encuestas a ramas productivas argentinas.

\section{Cuadro A3. Descripción de la muestra según variables estructurales}

\begin{tabular}{|l|c|c|c|c|c|c|c|c|}
\hline $\begin{array}{l}\text { Variables descriptivas } \\
\text { y de control. }\end{array}$ & $\begin{array}{c}\text { Maquinaria } \\
\text { agrícola }\end{array}$ & $\begin{array}{c}\text { Textil- } \\
\text { confecciones }\end{array}$ & $\begin{array}{c}\text { Servicios } \\
\text { petroleros }\end{array}$ & $\begin{array}{c}\text { Embarca- } \\
\text { ciones }\end{array}$ & $\begin{array}{c}\text { Auto- } \\
\text { motriz }\end{array}$ & Siderurgia & Morón & Total \\
\hline Ocupados (promedio) & 111.5 & 68.5 & 73.9 & 22.1 & 155.0 & 97.8 & 38.2 & 89.7 \\
\hline IED & 3.6 & 2.4 & 7.4 & - & 35.6 & 7.8 & 5.6 & 11.7 \\
\hline Grupo empresario & 6.0 & 5.0 & 22.2 & 19.2 & 41.0 & 23.0 & 11.4 & 19.8 \\
\hline $\begin{array}{l}\text { Coef. de exportación } \\
\text { (promedio). }\end{array}$ & 10.5 & 7.8 & 4.3 & 21.5 & 16.7 & 13.3 & 35.8 & 18.0 \\
\hline $\begin{array}{l}\text { Normas Iso } \\
\text { de aseguramiento } \\
\text { de la calidad. }\end{array}$ & 41.5 & 12.2 & 88.0 & 32.0 & 65.1 & 70.7 & 33.7 & 49.6 \\
\hline
\end{tabular}

Fuente: Elaboración propia con base en encuestas a ramas productivas argentinas. 


\begin{tabular}{|c|c|c|c|c|c|c|c|c|c|c|c|c|c|c|}
\hline $\begin{array}{l}\overline{\widetilde{D}} \\
\stackrel{0}{\circ}\end{array}$ & $\stackrel{0}{\dot{0}}$ & $\begin{array}{l}0 \\
\infty \\
0 \\
0\end{array}$ & 우 & $\underset{\tilde{m}}{\tilde{m}}$ & 옴 & $\underset{\infty}{\sim}$ & $\begin{array}{l}\text { nn } \\
\text { Q } \\
\end{array}$ & $\underset{\sim}{\stackrel{n}{N}}$ & $\overline{\grave{\lambda}}$ & $\bar{\sim}$ & นn & $\overline{\dot{m}}$ & $\hat{\stackrel{\infty}{\sigma}}$ & $\stackrel{m}{\dot{n}}$ \\
\hline $\begin{array}{l}\complement \\
\stackrel{\circ}{\circ} \\
\stackrel{0}{\Sigma}\end{array}$ & $\underset{\ddot{\theta}}{m}$ & $\begin{array}{l}\stackrel{+}{0} \\
\text { ¿े }\end{array}$ & 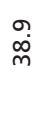 & $\begin{array}{l}\text { : } \\
\text { ì }\end{array}$ & $\stackrel{m}{m}$ & $\stackrel{\infty}{\stackrel{\infty}{\wedge}}$ & 号 & $\stackrel{\text { no }}{\stackrel{N}{N}}$ & ڤn & $\stackrel{\bar{\infty}}{\sim}$ & $\begin{array}{l}\infty \\
\text { ஸ் }\end{array}$ & $\underset{\infty}{\infty}$ & $\hat{ঙ}$ & 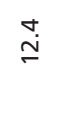 \\
\hline $\begin{array}{l}\frac{\pi}{0} \\
\frac{0}{2} \\
\frac{0}{0} \\
\frac{0}{0} \\
\text { in }\end{array}$ & $\stackrel{N}{0}$ & $\hat{o}$ & 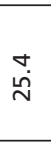 & $\stackrel{\sigma}{\sim}$ & $\overline{\dot{I}}$ & $\stackrel{\infty}{\infty}$ & $\begin{array}{l}\stackrel{0}{0} \\
\stackrel{0}{\circ}\end{array}$ & $\frac{\sigma}{m}$ & $\hat{\sigma}$ & $\begin{array}{l}0 \\
\infty \\
\infty\end{array}$ & $\stackrel{+}{\check{\sigma}}$ & $\stackrel{m}{\stackrel{m}{d}}$ & $\begin{array}{l}\circ \\
\stackrel{q}{q}\end{array}$ & : \\
\hline 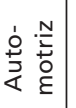 & $\underset{\infty}{\bar{\infty}}$ & $\stackrel{\sim}{\infty}$ & $\begin{array}{l}0 \\
\dot{0}\end{array}$ & $\stackrel{\sim}{\bar{N}}$ & $\stackrel{0}{\stackrel{0}{\digamma}}$ & $\begin{array}{l}m \\
\text { nn }\end{array}$ & 号 & $\begin{array}{l}m \\
\text { mे }\end{array}$ & $\stackrel{\sim}{\sim}$ & $\stackrel{\sim}{=}$ & $\bar{i}$ & $\begin{array}{l}\infty \\
\stackrel{\text { Dे }}{ }\end{array}$ & $\begin{array}{l}\stackrel{0}{n} \\
\text { ñ }\end{array}$ & 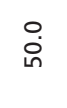 \\
\hline 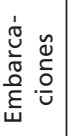 & $\hat{b}$ & $\stackrel{n}{\mathfrak{6}}$ & $\hat{\bar{y}}$ & $\stackrel{\circ}{\stackrel{\leftrightarrow}{~}}$ & $\overline{\bar{N}}$ & $\begin{array}{l}\tilde{N} \\
\hat{\sigma}\end{array}$ & $\begin{array}{l}\stackrel{0}{\circ} \\
\dot{\varphi}\end{array}$ & $\stackrel{\infty}{0}_{\infty}$ & $\begin{array}{l}\circ \\
\dot{q}\end{array}$ & $\begin{array}{l}\stackrel{\leftrightarrow}{d} \\
\dot{d}\end{array}$ & $\begin{array}{l}0 \\
\infty \\
\dot{\infty}\end{array}$ & $\begin{array}{l}\circ \\
\dot{m}\end{array}$ & $\begin{array}{l}\circ \\
\stackrel{\infty}{\sigma}\end{array}$ & $\begin{array}{l}\stackrel{0}{\varphi} \\
\dot{\varphi}\end{array}$ \\
\hline 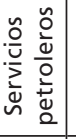 & $\frac{n}{\infty}$ & $\bar{i}$ & $\frac{9}{n}$ & $\underset{m}{m}$ & 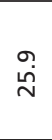 & $\begin{array}{l}\text { i. } \\
\text { }\end{array}$ & $\begin{array}{l}\text { mà } \\
\text { ñ }\end{array}$ & $\begin{array}{l}\infty \\
\dot{+}\end{array}$ & $\stackrel{\forall}{\underset{f}{+}}$ & $\underset{m}{m}$ & $\begin{array}{l}\stackrel{+}{*} \\
\dot{J}\end{array}$ & $\begin{array}{l}\infty \\
\dot{\Xi}\end{array}$ & $\hat{o}$ & $\begin{array}{l}\infty \\
\dot{\square}\end{array}$ \\
\hline 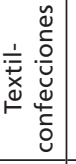 & $\begin{array}{l}\tilde{L} \\
\stackrel{\infty}{\infty}\end{array}$ & $\begin{array}{l}\text { Өે } \\
\text { ڤે }\end{array}$ & $\stackrel{\stackrel{N}{n}}{\check{n}}$ & $\underset{\substack{n \\
n}}{\stackrel{\infty}{n}}$ & $\overline{\dot{m}}$ & $\underset{\infty}{\dot{\infty}}$ & $\begin{array}{l}\text { nn } \\
\dot{\infty}\end{array}$ & $\stackrel{\leftrightarrow}{+}$ & $\begin{array}{c}m \\
\dot{g}\end{array}$ & $\begin{array}{l}\infty \\
\stackrel{\infty}{\sigma} \\
\infty\end{array}$ & $\stackrel{N}{\stackrel{n}{n}}$ & $\stackrel{N}{\text { in }}$ & $\begin{array}{l}\infty \\
\infty \\
\infty \\
\sigma\end{array}$ & $\stackrel{\simeq}{\simeq}$ \\
\hline 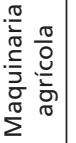 & $\begin{array}{l}\text { வ் } \\
\text { ه }\end{array}$ & 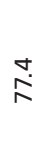 & คุ. & $\underset{\sim}{\tilde{F}}$ & $\stackrel{m}{\infty}$ & $\begin{array}{l}m \\
\text { gे }\end{array}$ & $\underset{\infty}{\stackrel{0}{\infty}}$ & $\stackrel{m}{m}$ & $\stackrel{\text { ti }}{\text { in }}$ & $\begin{array}{l}0 \\
\stackrel{\gamma}{\forall}\end{array}$ & í & 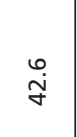 & $\hat{\emptyset}$ & $\stackrel{\varphi}{\dot{\varphi}}$ \\
\hline \multirow[t]{2}{*}{ 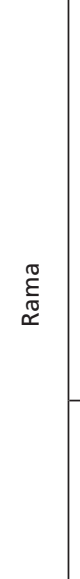 } & 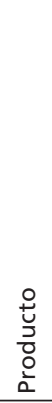 & 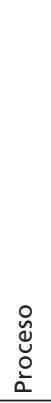 & 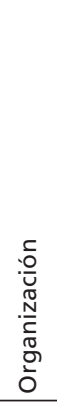 & 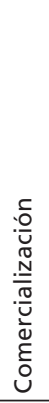 & 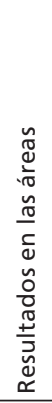 & 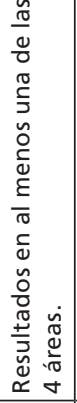 & 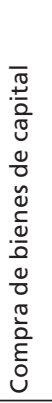 & 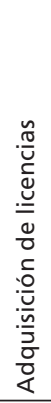 & 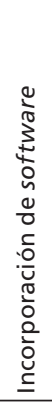 & 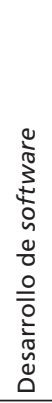 & 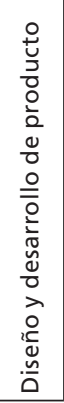 & 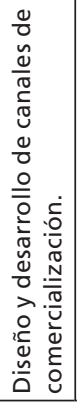 & 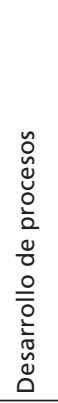 & 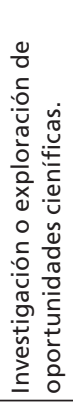 \\
\hline & \multicolumn{6}{|c|}{ 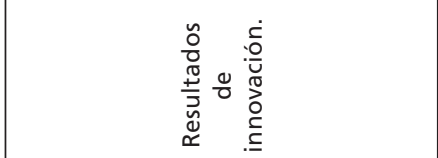 } & \multicolumn{8}{|c|}{ 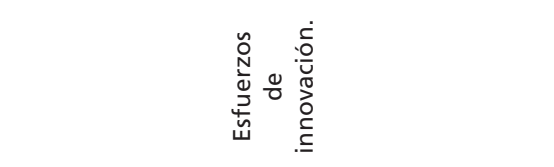 } \\
\hline
\end{tabular}




\begin{tabular}{|c|c|c|c|c|c|c|c|c|c|c|c|c|c|c|c|}
\hline $\begin{array}{l}\bar{\pi} \\
\stackrel{0}{0}\end{array}$ & $\stackrel{\sim}{\stackrel{\sim}{n}}$ & 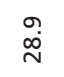 & $\overline{\dot{m}}$ & ֻั & $\begin{array}{l}\infty \\
\stackrel{m}{\rho}\end{array}$ & $\stackrel{\infty}{\tilde{N}}$ & $\stackrel{\mathscr{\vartheta}}{\text { ษ }}$ & $\stackrel{\circ}{m}$ & $\underset{\sim}{\stackrel{\sim}{\sim}}$ & $\begin{array}{c}N \\
\infty \\
0\end{array}$ & 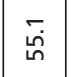 & $\begin{array}{l}9 \\
\dot{g} \\
\dot{g}\end{array}$ & $\stackrel{\stackrel{n}{N}}{\wedge}$ & $\stackrel{\sim}{\sim}$ & $\underset{+}{+}$ \\
\hline $\begin{array}{l}\text { ¿ } \\
\stackrel{0}{0} \\
\sum\end{array}$ & $\begin{array}{l}\circ \\
\dot{m} \\
\dot{m}\end{array}$ & бே & 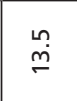 & $\bar{g}$ & $\begin{array}{l}\stackrel{0}{\sim} \\
\stackrel{\dot{n}}{n}\end{array}$ & $\stackrel{\circ}{\stackrel{\leftrightarrow}{ }}$ & $\underset{N}{N}$ & $\begin{array}{l}\stackrel{\sigma}{\infty} \\
\infty\end{array}$ & $\underset{\infty}{\sigma}$ & $\begin{array}{l}\tilde{Z} \\
\tilde{G}\end{array}$ & 일 & O̊ & 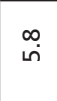 & $\dot{f}$ & $\stackrel{\infty}{0}$ \\
\hline $\begin{array}{l}\frac{\pi}{0} \\
\frac{\pi}{3} \\
\frac{2}{0} \\
\frac{0}{0} \\
\text { in }\end{array}$ & 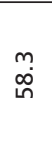 & $\stackrel{\sim}{\dot{N}}$ & 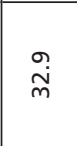 & $\begin{array}{l}\stackrel{9}{\dot{\gamma}} \\
\stackrel{\leftrightarrow}{n}\end{array}$ & $\begin{array}{l}\stackrel{\operatorname{n}}{\infty} \\
\stackrel{\infty}{\sigma}\end{array}$ & $\stackrel{\circ}{\stackrel{\circ}{=}}$ & $\frac{N}{m}$ & $\begin{array}{l}\infty \\
\dot{\sigma}\end{array}$ & $\overline{\grave{N}}$ & ホ̀ & $\begin{array}{l}0 \\
\ddot{6}\end{array}$ & $\frac{0}{6}$ & $\stackrel{\leftrightarrow}{\leftarrow}$ & ' & $\hat{i}$ \\
\hline 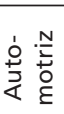 & $\begin{array}{c}\bar{\infty} \\
\infty \\
\infty\end{array}$ & $\stackrel{m}{i}$ & $\frac{\circ}{\infty}$ & $\begin{array}{l}\dot{0} \\
\stackrel{\text { in }}{n}\end{array}$ & $\stackrel{\hat{i}}{\text { in }}$ & $\underset{\substack{\infty \\
m}}{+}$ & $\stackrel{\text { 물 }}{=}$ & Фి & $\begin{array}{l}0 \\
\infty \\
i \\
i\end{array}$ & $\underset{\infty}{\dot{\infty}}$ & $\frac{\varphi}{\infty}$ & $\begin{array}{l}0 \\
0 \\
\infty \\
\infty\end{array}$ & $\stackrel{\sim}{\infty}$ & $\bar{\sigma}$ & $\stackrel{\sim}{\longrightarrow}$ \\
\hline 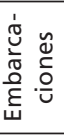 & 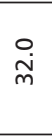 & @̊ & ' & $\stackrel{\sim}{\infty}$ & $\underset{\infty}{m}$ & 芦 & กุ & $\stackrel{\sim}{\circ}$ & $\stackrel{\stackrel{n}{=}}{=}$ & $\overline{\tilde{N}}$ & $\overline{\ddot{v}}$ & $\bar{\sim}$ & & $\stackrel{\circ}{\dot{\sigma}}$ & ' \\
\hline 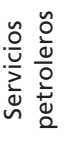 & $\bar{m}$ & $\hat{\dot{q}}$ & $\stackrel{\sim}{N}$ & $\stackrel{\circ}{\stackrel{+}{J}}$ & 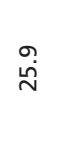 & 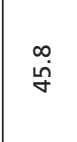 & ஸ̊. & $\bar{\infty}$ & $\stackrel{\sim}{\stackrel{N}{N}}$ & m. & $\stackrel{\sim}{\stackrel{\infty}{\infty}}$ & $\stackrel{0}{\ddot{\theta}}$ & $\stackrel{\infty}{m}$ & $\begin{array}{l}\infty \\
m\end{array}$ & ' \\
\hline 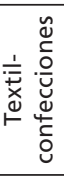 & $\check{\check{\Sigma}}$ & $\stackrel{\sim}{\simeq}$ & $\begin{array}{l}\bullet \\
\dot{J}\end{array}$ & g̣̊ & $\begin{array}{l}\stackrel{0}{0} \\
\stackrel{m}{m}\end{array}$ & $\stackrel{\curvearrowright}{\curvearrowright}$ & $\overline{\dot{b}}$ & $\begin{array}{l}\dot{0} \\
\dot{m} \\
\dot{m}\end{array}$ & $\stackrel{m}{\sim}$ & $\begin{array}{c}\infty \\
\infty \\
\stackrel{\infty}{\sigma}\end{array}$ & ڤ્n & $\overline{\dot{m}}$ & $\overline{i n}$ & $\stackrel{n}{r}$ & $\stackrel{\leftrightarrow}{\sim}$ \\
\hline 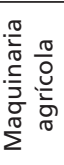 & $\begin{array}{l}0 \\
\text { மُ }\end{array}$ & $\underset{\dot{J}}{\bar{J}}$ & $\stackrel{\circ}{\stackrel{\circ}{\circ}}$ & $\overline{\dot{g}}$ & $\begin{array}{l}\text { ọ } \\
\text { in }\end{array}$ & $\bar{\sim}$ & $\begin{array}{l}\text { டி } \\
\text { ஜn }\end{array}$ & $\stackrel{\sim}{\infty}$ & $\stackrel{+}{\oplus}$ & $\begin{array}{l}\circ \\
\dot{\infty}\end{array}$ & $\begin{array}{l}\stackrel{0}{\mathscr{\gamma}} \\
\stackrel{\dot{\gamma}}{ }\end{array}$ & 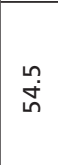 & $\stackrel{\stackrel{n}{+}}{\stackrel{N}{d}}$ & $\stackrel{\sim}{\sim}$ & 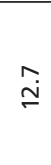 \\
\hline$\underset{\substack{\pi \\
\simeq}}{\stackrel{\pi}{\varepsilon}}$ & 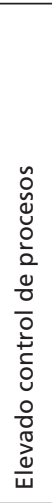 & 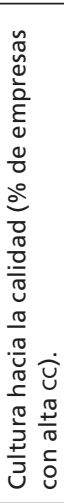 & 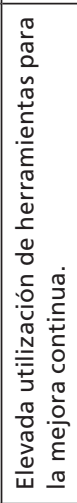 & 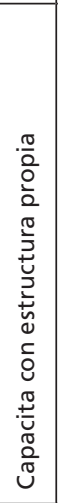 & 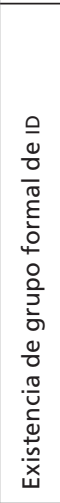 & 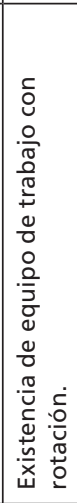 & 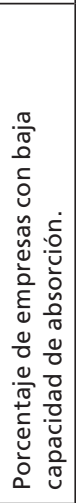 & 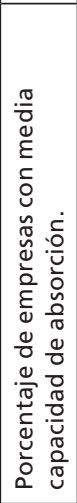 & 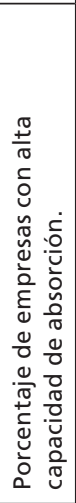 & 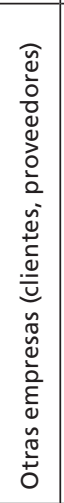 & 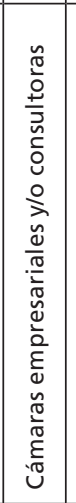 & 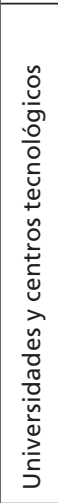 & 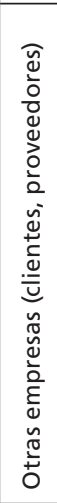 & 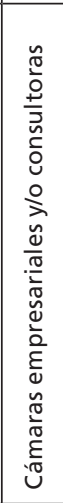 & 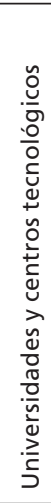 \\
\hline & \multicolumn{6}{|c|}{ 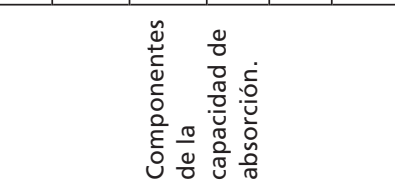 } & \multicolumn{3}{|c|}{ 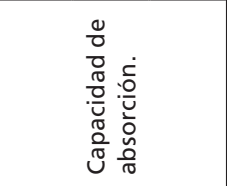 } & \multicolumn{3}{|c|}{ 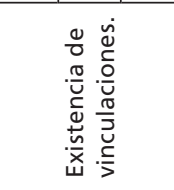 } & \multicolumn{3}{|c|}{ 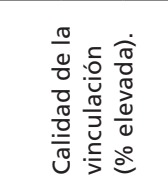 } \\
\hline
\end{tabular}




\section{REFERENCIAS BIBLIOGRÁFICAS}

Acemoglu, D. (2002), "Technical Change, Inequality, and the Labor Market”, Journal of Economic Literature 40 (1), pp. 7-72.

Ahuja, Gautam (2000), "Collaboration Networks, Structural Holes and Innovation: a Longitudinal Study", Administrative Science Quarterly, Vol. 45, núm. 3, pp. 425-455

Albornoz, F., y G. Yoguel (2004), "Competitiveness and Production Network: the Case of the Argentine Automotive Sector", Industrial and Corporate Change, Vol 13, núm. 4.

Antonelli, C. (1999), "The Evolution of Industrial Organization of the Production of Knowledge", Cambridge Journal of Economics, Vol. 23.

(2008), Localised Technological Change. Towards the Economics of Complexity, Londres y Nueva York, Ed. Routledge.

Arthur, B. (1999), “Complexity and the Economy”, Sciences, núm. 2.

Becattini, G. (1989), Modelli locali di sviluppo, Mulino, Bologna.

Benavente, J. M., y R. Lauterbach (2008), “Technological Innovation and Employment: Complements or Substitutes?, European Journal of Development Research, Taylor and Francis Journals, Vol. 20 núm. 2, pp. 318-329.

Berman, E., J. Bound, y Griliches (1994), "Changes in the Demand for Skilled Labor within US Manufacturing: Evidence from the Annual Survey of Manufacturers", The Quarterly Journal of Economics, 109 (2), pp. 367-397.

Bisang, Roberto, Marta Novick, Sebastián Sztulwark, y Gabriel Yoguel (2005), "Las redes de producción y el empleo", en Mónica Casalet, Mario Cimoli y Gabriel Yoguel (comps.), Redes, jerarquías y dinámicas productivas, Buenos Aires, Miño y Dávila, OIT-Flacso, México.

Brouwer, E., A. Kleinknecht, y J.O.N. Reijnen (1993), "Employment Growth and Innovation at the Firm Level. An Empitical Study”, Evolutionary Economics, Springer-Verlag.

Caloghirou, Yannis, Ioanna Kastelli, y Aggelos Tsakanikas (2004), "Internal Capabilities and External Knowledge Sources: Complements or Substitutes for Innovative Performance?", Technovation, Vol. 24, núm. 1, pp. 29-39.

Camagni, R. (1991), Innovation Networks: Spatial Perspectives, Londres, Belhaven Press.

Chennels, L., y J. Van Reenen (1999), "Has Technology Hurt Less Skilled Workers", Institute for Fiscal Studies, Londres, Working Paper Series, núm. W99/27.

Cohen, W. M., y D. Levinthal (1989), "Innovation and Learning: The Two Faces of R\&D”, The Economic Journal, Vol. 99, núm. 397, septiembre. 
Cohen, W. M., y Daniel A. Levinthal (1990), “Absorptive Capacity: A New Perspective on Learning and Innovation Journal”, Administrative Science Quarterly, Vol. 35 núm. 1, pp. 128-152

Coombs, Rod, y J. Metcalfe Stan (2000), Universities, the Science Base and the Innovation Performance of the UK, CRIC Briefing Paper, núm. 5. http://www.cric.ac. uk/cric/Pdfs/bp5.pdf

Coriat, B. (1992), El taller y el robot. Ensayos sobre el fordismo y la producción en masa en la era de la electrónica, Buenos Aires, Siglo XXI Editores.

, y O. Weinstein (2002), "Organizations, Firms and Institutions in the Generation of Innovation”, Research Policy, 31 (2), pp. 273- 290.

Cullen, P. A. (2000), “Contracting, cooperative relations and extended enterprises", Technovation. Vol. 20, núm. 7, pp. 363-372.

Dosi, G., (1991). "Some Thoughts on the Promises, Challenges and Dangers of an Evolutionary Perspective in Economics", Journal of Evolutionary Economics, primavera, Vol. 1, núm. 1, pp. 5-7.

__ y Y. Kaniovski (1994), “On "Badly Behaved” Dynamics”, Journal of Evolutionary Economics, núm. 4, pp. 93-123.

— y Nelson, Richard R (1994), “An Introduction to Evolutionary Theories in Economics”, Journal of Evolutionary Economics, primavera, Vol. 4, núm. 3, pp. 153-172.

Edquist, C. (2000), Systems of Innovation: Growth, Competitiveness and Employment, MD McKelvey, E. Elgar Pub, Reino Unido, Cheltenham.

Erbes, A., V. Robert, G. Yoguel, J. Borello, y V. Lebedinsky (2006), “Regímenes tecnológicos, de conocimiento y competencia en diferentes formas organizacionales: la dinámica entre difusión y apropiación”, Revista Desarrollo Económico, Vol. 46, núm. 181, Buenos Aires, abril-junio.

E. Tacsir, y G. Yoguel (2008), “Endogenous Competences and Linkages Development", ponencia presentada en la 6ta Conferencia Internacional Globelics, del 22 al 24 septiembre, ciudad de México.

- V. Robert, y G. Yoguel (2010), "Capacities, Innovation and Feedbacks in Production Networks in Argentina", Economics of Innovation and New Technolo$g y$, Londres, Routlege, en prensa.

Evangelista, R., y M. Savona, (2001), Innovation, Employment and Skills in Services. Firm and Sectoral Evidence, documento presentado en la ECIS Conference The Future of Innovation Studies Eindhoven, Center for Innovation Studies, Países Bajos, 20-23 de septiembre.

Gereffi, G., J. Humphrey, y T. Sturgeon (2005), “The Governance of Global Value Chains”, Review of International Political Economy, Vol. 12, núm. 1. 
Grandori, A., y G. Soda (1995), Interfirm Networks: Antecedents, Mechanisms and Forms, Organization Studies Sage. Vol. 16., núm. 2, pp. 183-214.

Hall, B., F. Lotti y J. Mairesse (2008), “Employment, Innovation, and Productivity: Evidence from Italian Microdata", Industrial and Corporate Change, Vol. 17, núm. 4. pp. 813-839.

Humphrey, J., y H. Schmitz (2000), "Governance and Upgrading: Linking Industrial Cluster and Global Value Chain Research", IDS Working Paper, núm. 120, University of Sussex.

Jaumandreu, J. (2003), “Does Innovation Spur Employment? A Firm-Level Analysis Using Spanish CIS Data", Documento de Trabajo, Universidad Carlos III de Madrid. http://www.eco.uc3m.es/temp/jaumandreu.pdf

Laursen, Keld, y Salter Ammon (2004), "Searching High and Low: What Types of Firms Use Universities as a Source of Innovation?”, Research Policy, núm. 33, pp. 12011215.

Malerba, F., y L. Orsenigo (1993), “Technological Regimes and Firm Bebavior”, Industrial and Corporate Change, Vol. 2, núm. 1, pp. 45-71.

- y - (1997), "Technological Regimes and Sectoral Patterns of Innovative Activities", Industrial and Corporate Change, Vol. 6, núm. 1.

Metcalfe, S., J. Foster, y R. Ramlogan (2006), “Adaptive Economic Growth”, Cambridge Journal of Economics, Vol. 30, núm. 1, pp. 7-32

Mowery, David, Joanne Oxley, y Brian Silverman (1996), "Strategic Alliances and Interfirm Knowledge Transfer”, Strategic Management Journal, Vol. 17, núm. especial, invierno, pp. 77-91.

Narula (2001), “Understanding Absorptive Capacities in an 'Innovation Systems' Context: Consequences for Economic and Employment Growth DRUID”, Working Paper, núms. 04-02

Nelson, R., y S. G. Winter (1982), An Evolutionary Theory of Economic Change, Cambridge, Harvard University Press.

Norman, P. (2002), "Protecting Knowledge in Strategic Alliances. Resource and Relational Characteristic", The Journal of High Technology Management Research, Vol. 13, núm. 2.

Novick, M., S. Rojo, y G. Yoguel (2009), The Complex Relationship Between Innovation and Employment, trabajo presentado en el Seminario Eulaks, Viena, junio.

Pavitt, K. (1984), "Sectorial Patterns of Technical Change: Towards a Taxonomy and a Theory”, Research Policy, núm. 13. 
Peters, B. (2005), "Employment effects of different innovation activities: Microeconometric evidence". ZEW - Centre for European Economic Research, Documento de Trabajo núm. 04-073. http://www.socialpolitik.de/tagungshps/2005/Papers/ Peters.pdf

Petit, Pascal (1998), "Employment and Technological Change”, en Stoneman (comp.), Handbook of the Economics of Innovation and Technological Change, Oxford, Reino Unido, Blackwell.

Pianta, M. (2006), “Innovation and employment”, en Fagerberg Jan, David C. Mowery, y Richard R. Nelson (comps.), Oxford Handbook of Innovation, Oxford, Oxford University Press.

Piva, Mariacristina, Enrico Santarelli, y Marco Vivarelli (2005), “The Skill Bias Effect of Technological and Organisational Change: Evidence and Policy Implications", Research Policy, Elsevier, Vol. 34 núm. 2, pp. 141-157.

Powell, W.W., y S. Grodal (2005), "Networks of Innovators", The Oxford Handbook of Innovation, Fagerberg, Mowery y Nelson (comps.), Oxford University Press, pp. 56-85.

Richardson, George (1972), “The Organization of Industry”, The Economic Journal, Vol. 82, núm. 327, pp. 883-896.

Rivera Ríos, M. A., V. Robert, y G. Yoguel (2009), “Cambio tecnológico, complejidad e instituciones: una aproximación desde la estructura industrial e institucional de Argentina y México", Problemas del desarrollo, Instituto de Investigaciones Económicas, México, UNAM.

Roitter S, A. Erbes, G. Yoguel, M. Delfini, y A. Pujol (2007), “Conocimiento, organización del trabajo y empleo en agentes pertenecientes a las tramas productivas automotriz y siderúrgica", Revista Economía: Teoría y Práctica, núm. 26, México, UAM-Iztapalapa.

Schmitz, H. (1995), "Collective Efficiency: Growth Path for Small-Scale Industry", Journal of Development Studies, Vol. 31, núm. 4, pp. 529-566.

— y K. Nadvi (1999), "Clustering and Industrialization: Introduction", World Development, Reino Unido, Elsevier Science, Vol. 27, núm. 9, pp. 1503-1514.

Santoro, Michael, y Shanthi Gopalakrishnan (2000), "The Institutionalization of Knowledge Transfer Activities within Industry-University Collaborative Ventures", Journal of Engineering and Technology Management, Vol. 17, núms. 3-4, pp. 299-319.

Stoneman P. (1995), Handbook of the Economics of Innovation and Technological Change, Oxford, Reino Unido, Blackwell Handbooks in Economics, Blackwell. 
Teece, David (1992), “Competition, Cooperation, and Innovation. Organizational Arrengements for Regimes of Rapid Technological Progress", Journal of Economic Behaviour \& Organization, núm. 18, pp. 1-25.

Tether, B. (2000), "Small Firms, Innovation and Employment Creation in Britain and Europe: A question of expectations...", Londres, Pergamon- Elseiver, Technovation, Vol. 20, núm. 2, pp. 109-113.

Van den Bosch, A. J. Frans, W. Henk, Volberda y Michiel de Boer (1999), "Coevolution of Firm Absorptive Capacity and Knowledge Environment: Organizational Forms and Combinative Capabilities", Organization Science, Vol. 10, Núm. 5, Focused Issued: Coevolution of Strategy and New Organizational Forms, pp. 551-568.

Van Reenen, J. (1997), "Employment and Technological Innovation: Evidence from U.K. Manufaturing Firms", Journal of Labor Economics, The University of Chicago Press, Vol. 15, núm.2, pp. 255-284.

Vivarelli, Evangelista, y Pianta (1996), "Innovation and Employment in Italian Manufacturing Industry”, Research Policy, núm. 25, pp. 1013-1026.

Yoguel, Gabriel (2007), “Tramas productivas y generación de ventajas competitivas: un abordaje metodológico para pasar de la empresa individual a la red", en Novick Marta y Héctor Palomino (comps.), Estructura productiva y empleo, Buenos Aires, Miño y Dávila, Ministerio de Trabajo.

(2009), Redes de conocimiento en tramas productivas argentinas: Generación, circulación y apropiación de conocimiento y creación de ventajas competitivas, México, IDRC-Flacso, en prensa.

Zahra, Shaker, y Gerard George (2002), “Absorptive Capacity; a Review Reconceptualization, and Extension”, Academy of Management Review, Vol. 27, núm. 2, pp. 185-203. 